\title{
Robust estimation and diagnosis of wind turbine pitch misalignments at a wind farm level
}

\author{
Ester Sales-Setién ${ }^{1 \star}$ and Ignacio Peñarrocha-Alós ${ }^{1}$ \\ ${ }^{1}$ Department of Industrial Engineering and Design, Universitat Jaume I, Castellón, Spain \\ *esales@uji.es
}

\begin{abstract}
Wind turbine pitch misalignments provoke aerodynamic asymmetries which cause severe damage to the turbine. Hence, it is of interest to develop fault tolerant strategies to cope with pitch misalignments. Fault tolerant strategies require the information regarding the diagnosis and the estimation of the faults. However, most existing works focus only on open-loop misalignment diagnosis and do not provide robust fault estimates. In this work, we present a novel strategy to both estimate and diagnose pitch misalignments. The proposed strategy is developed at a wind farm level and it exploits altogether the information provided by the temporal and spatial relations of the turbines in the farm. Fault estimation is first addressed with a closed-loop switched observer. This observer is robust against disturbances and it adapts to the varying conditions along the wind turbine operation range. Fault diagnosis is then achieved via statistical-based decision mechanisms with adaptive thresholds. Both the observer and the decision mechanisms are designed to guarantee the desired performance. Introducing some restrictions over the number of simultaneous faulty turbines in the farm, the proposed approach is ameliorated via a bank of the aforementioned observers and decision mechanisms. Finally, the strategies are tested using a well-known wind farm benchmark.
\end{abstract}

Keywords: Wind turbine pitch system, fault estimation, fault diagnosis, observer, optimisation.

\section{Introduction}

Wind energy has grown in importance over the last two decades as it has proven to be a promising and powerful source of renewable energy [1]. However, the maintainability and reliability of wind turbines (WTs) is still a challenging and critical issue due to the high costs associated to maintenance operations [2]. The issues related to the pitch system are of particular interest to the wind industry due to their long downtimes and high failure rates [2,3]. These issues include actuator, sensor and imbalance faults [4-6]. A common pitch imbalance fault is aerodynamic asymmetry, which may be caused by the misalignment of one or more blades. This misalignment can be originated by several factors, including high wind shear and manufacturing, installation or control errors [6,7]. In general, aerodynamic asymmetries represent a significant problem for WTs, as also witnessed by the fact that certification uidelines require relatively small pitch misalignments [8]. When a pitch misalignment is present, the loadings experienced by the blades are not balanced which results in vibrations that negatively affect other components of the turbine. Moreover, the power quality is degraded and the power generation efficiency is reduced [7].

It is widely recognised that pitch actuator and sensor faults can be dealt with at the WT level. A well-established benchmark for actuator and sensor fault diagnosis (FD) in WTs was presented in [9]. Several solutions have been proposed showing the effectiveness of FD for predictive maintenance, e.g. [10-14]. However, imbalance faults are more challenging. As stated by [6], most exiting methods for imbalance fault diagnosis require additional sensors such as vibrational sensors $[15,16]$. This extra equipment is inevitably subject to failures and it is generally difficult to access. Thus, current-based imbalance diagnosis methods have gained more attention [6,7]. However, as recognised by [6], there are intrinsic challenges in using current signals for imbalance diagnosis: the characteristic frequencies of imbalance faults depend on the shaft rotating frequency and the useful information in current signals for FD is characterized by a low signal-to-noise ratio. 
Another approach is to diagnose imbalance faults at a wind farm (WF) level, where each WT is considered in comparison to other WTs in the WF. In [5], a WF data-mining approach was developed for monitoring blade imbalance faults. Motivated by this work, a new benchmark has been presented in [17] for FD in WTs at a WF level. Fewer solutions to this problem can be found in the bibliography [18-22]. The FD strategies of these works are based on two kinds of residuals, which are signals representing inconsistencies. On the one hand, temporal residuals represent the inconsistencies between the pitch model output and the pitch measurement of a single WT; on the other hand, spatial residuals are used to represent the inconsistencies between the pitch measurements of different WTs. Borcehrsen et al. [18] utilize open-loop temporal residuals in dynamical cumulative sums. Alternatively, Duviella et al. [20] present a FD approach based on spatial residuals and Blesa et al. [19] compute both spatial and open-loop temporal residuals via nonlinear parameter varying parity equations. For its part, Simani et al. [21,22] present fuzzy and neural network techniques to achieve FD.

The pitch model of a WT is affected by various non-negligible disturbances: wind speed (WS) estimation errors, turbulences and measurement noises [17]. Hence, the open-loop temporal residuals in [18,19] and the spatial

\section{residuals in [19,20] may be significantly distubed. It is thus of interest to develop closed-loop strategies with} a better performance w.r.t. disturbances. Besides this issue, the residual-based techniques in [18-21] focus on obtaining the information about the appearance and the location of a fault (fault diagnosis or FD). However, the information about the size and shape of a fault (fault estimation or FE) is also of paramount importance for both real-time decisions and active fault tolerant control (AFTC) [23,24]. Rather than on separate spatial and temporal residuals, an optimal FE strategy should be based on a systematic approach that automatically merges all the information acquired from both temporal and spatial inconsistencies.

In this work, we thus propose a spatially and temporally integrated closed-loop strategy for the estimation and diagnosis of pitch misalignments. First, a multi-input multi-output (MIMO) model-based augmented observer is used for FE [25,26]. Provided that the disturbances affecting the pitch system depend on the WT operation conditions, a constant augmented observer may lead to too conservative results. Hence, we develop instead a switched augmented observer for FE [27] and we design it via an optimization problem that fixes the trade-off between the cumulative squared error experienced by the estimates due to faults and the root mean square of the estimates due to noises and uncertainties. Providing this systematic performance-based optimal approach for tuning the FE observer is an advantage when compared to other design algorithms, where some user expertise is necessary. For instance, numerical extensive simulations and trial and error procedures are necessary to tune the algorithms in [22]. Second, we feed the fault estimates provided by the proposed observer into statistical-based decision mechanisms to achieve FD [28]. Constant thresholding does not properly adapt to the time-varying properties of the estimates provided by the switched observer. Therefore, we propose to compute adaptive thresholds using the False Alarm Rate (FAR) criterion. When compared to the constant thresholds in [18,22], the proposed adaptive thresholds enhance a tighter adjustment to the fault estimates leading to better fault diagnosability.

The rest of this paper is organized as follows. Section 2 gives the problem formulation. Section 3 presents the pitch misalignment model on which the proposed FE and FD strategy is based. Section 4 presents and characterizes an open-loop estimation strategy of the inputs of the system and Section 5 develops and designs a closed-loop FE estimation strategy for pitch misalignments. Then, Section 6 utilizes the resulting fault estimates to build and design a FD strategy. In Section 7 we extend the proposed FE and FD strategy for cases with some restrictions regarding the number of simultaneous faulty WTs in the WF. Simulation results are reported in Section 9 to show the effectiveness of the proposed approach, followed by some concluding remarks in Section 10.

Notation: Let $A$ and $B$ be some matrix and $a$ be some vector. $A[i, j]$ denotes the element in the $i$-th row and $j$-th column of $A$ and $a[i]$ denotes the $i$-th element in $a$. $A \preceq 0$ means that $A$ is negative semidefinite and similar 85 applies to $\succeq$. The trace of matrix $A$ is represented as $\operatorname{tr}(A)$. Let $x_{k}$ be a vector of stochastic signals at a sample $k$. We write $\left\|x_{k}\right\|_{\infty} \triangleq \max _{i}\left|x_{k}[i]\right|$ for the $\max$ norm of vector $x_{k}$. We write $\|x\|_{\infty} \triangleq \max _{k} \max _{i}\left|x_{k}[i]\right|$ for the $l_{\infty}$ norm of signal $x,\|x\|_{2}^{2} \triangleq \lim _{K \rightarrow \infty} \sum_{k=1}^{K} x_{k}^{T} x_{k}$ for its $l_{2}$ norm and $\|x\|_{\mathrm{RMS}}^{2}=\frac{1}{K}\|x\|_{2}^{2}$ for its RMS norm. 


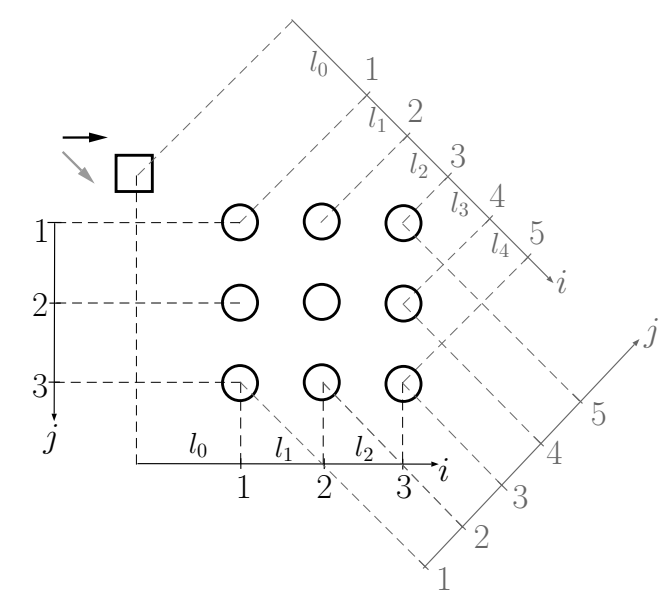

Figure 1. Layout of the WF benchmark ( $\bigcirc$ : WT, $\square$ : Wind mast, $\rightarrow$ : Wind direction). Black: $0^{\circ}$ layout, Gray: $45^{\circ}$ layout.

Expected value, probability and absolute value are denoted by $\mathbb{E}\{\cdot\}, \mathbb{P}\{\cdot\}$ and $|\cdot| . I_{n}$ is the identity matrix of size $n \times n, \mathbf{1}_{n \times m}$ is a matrix of ones of size $n \times m$ and 0 is the zero matrix of appropriate dimensions.

\section{Problem Statement}

Consider the WF benchmark [17] consisting of $N=9$ WTs of $4.8 \mathrm{MW}$. The benchmark studies two different wind directions: $0^{\circ}$ and $45^{\circ}$. The WTs can be thus named according to the wind direction as shown in Fig. 1. We consider that the wind is perpendicular to the rows of WTs (numbered as $i=1, \ldots, n_{i}$ ) and parallel to the columns of WTs (numbered as $j=1, \ldots, n_{j}$ ). There is a wind mast at row $i=0$. We denote the distance between two consecutive rows $i$ and $i+1$ as $l_{i}$. For the $0^{\circ}$ layout, we have $l_{0}=1150 \mathrm{~m}$ and $l_{i}=1138.44 \mathrm{~m}$ for all $i \neq 0$.

The WS acting on the blades of each WT $(i, j)$ is composed by the mean WS acting on the WTs in the $i$-th row, denoted as $\nu^{i}$, and a zero-mean turbulence component of known variance $\left(\sigma_{t}^{2}=0.2 \mathrm{~m}^{2} / \mathrm{s}^{2}\right)$, denoted as $\tilde{\nu}_{t}^{i, j}$, i.e.,

$$
\nu^{i, j}=\nu^{i}+\tilde{\nu}_{t}^{i, j}
$$

Hence, we deduce that all the WTs $(i, j)$ in a row $i\left(j=1, \ldots, n_{j}\right)$ operate under similar wind conditions. Neither the effective WS nor the mean WS are measured; only the measurement of the mean WS at the wind mast is available: $\hat{\nu}^{0}=\nu^{0}+\tilde{\nu}^{0}$, with $\nu^{0}$ being the WS at the wind mast, $\hat{\nu}^{0}$ being its measurement and $\tilde{\nu}^{0}$ being the corresponding sensor noise.

The collective pitch system of each WT $(i, j)$ can be modelled as a first order closed-loop system between the collective pitch angle (henceforth, the collective pitch), named after $\beta^{i, j}$, and the collective pitch reference, named after $\beta_{r}^{i, j},[29]$ :

$$
\dot{\beta}^{i, j}=\tau_{\beta}\left(\beta_{r}^{i, j}-\beta^{i, j}\right)
$$

where $\tau_{\beta}$ is a known transfer function coefficient $\left(\tau_{\beta}=1.6 \mathrm{rad} / \mathrm{s}\right)$. The collective pitch reference $\beta_{r}^{i, j}$ is a nonlinear function $g(\cdot)$ of the actual power reference, denoted as $P_{r}$, and the effective WS acting on the blades of the turbine $(i, j)$, denoted as $\nu^{i, j}$ :

$$
\beta_{r}^{i, j}=g\left(\nu^{i, j}, P_{r}\right)
$$

see Fig.2 (values borrowed from the look up tables in [17]). In this work, we assume that the actual power reference, $P_{r}$, is known because it can be deduced from the variables provided by the WF controller (see Remark 1). For its part, the effective WS, $\nu^{i, j}$, is not known and it must be estimated from the WS measurement at the wind mast, $\hat{\nu}^{0}$, using certain propagation method (see Section 4). 


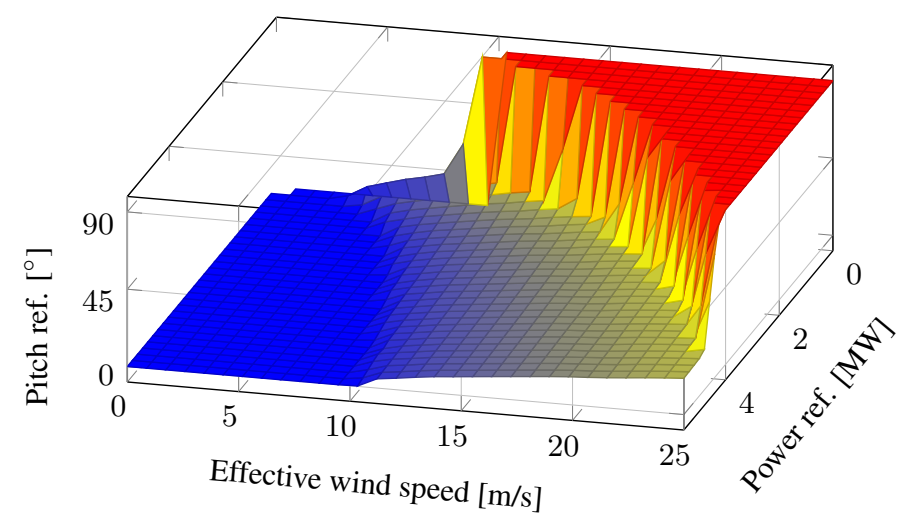

Figure 2. Nonlinear function $g\left(\nu^{i, j}, P^{r}\right)$.

\begin{tabular}{cllc}
\hline Variable & Description & Location & Units \\
\hline$\beta^{i, j}$ & Collective pitch (Collective pitch angle) & WT $(i, j)$ & {$\left[{ }^{\circ}\right]$} \\
$\beta_{r}^{i, j}$ & Collective pitch reference & WT $(i, j)$ & {$\left[{ }^{\circ}\right]$} \\
$y^{i, j}$ & Collective pitch measurement & WT $(i, j)$ & {$\left[{ }^{\circ}\right]$} \\
$w^{i, j}$ & Collective pitch measurement noise & WT $(i, j)$ & {$\left[^{\circ}\right]$} \\
$f^{i, j}$ & Offset caused by pitch misalignment & WT $(i, j)$ & {$\left[{ }^{\circ}\right]$} \\
$P_{r}$ & WT actual power reference & WT $(i, j), i=1, \ldots, n_{i}, j=1, \ldots, n_{j}$ & {$[\mathrm{MW}]$} \\
$P_{t}$ & WT static power reference & WT $(i, j), i=1, \ldots, n_{i}, j=1, \ldots, n_{j}$ & {$[\mathrm{MW}]$} \\
$P_{f}$ & WF static power reference & WF & {$[\mathrm{MW}]$} \\
$\nu^{i, j}$ & Effective wind speed & WT $(i, j)$ & {$[\mathrm{m} / \mathrm{s}]$} \\
$\nu^{i}$ & Mean wind speed in the $i$-th row & WT $(i, j), i=1, \ldots, n_{i}$ & {$[\mathrm{~m} / \mathrm{s}]$} \\
$\tilde{\nu}_{t}^{i, j}$ & Wind turbulence component & WT $(i, j)$ & {$[\mathrm{m} / \mathrm{s}]$} \\
\hline
\end{tabular}

Table 1. Variables involved in the collective pitch system model of the WT $(i, j)$.

Remark 1. The network operator determines the WF power demand that ensures a reliable connection of the WF to the electrical grid $^{1}$. Then, the WF controller computes the WF static power reference, denoted as $P_{f}$, as a function of the WF power demand and the actual WF power generation ${ }^{2}$. The WT static power reference, denoted 115 as $P_{t}$, is obtained from dispatching $P_{f}$ among the WTs in the WF. In this work, we consider that $P_{t}=P_{f} / N$. Finally, $P_{r}$ is deduced from the WT dynamic behaviour to changes in $P_{t}$. In the WF benchmark [17], it satisfies $\dot{P}_{r}=\tau_{p}\left(P_{t}-P_{r}\right)$ with $\tau_{p}=1.2 \mathrm{rad} / \mathrm{s}$, i.e., $P_{r}$ is a smoothed version of $P_{t}$.

The misalignment of one or more blades of the WT $(i, j)$ can be modelled as an additive fault, denoted as $f^{i, j}$, affecting the collective pitch measurement, denoted as $y^{i, j}$. The measurement $y^{i, j}$ is also affected by a zero-mean Gaussian sensor noise of known variance $\left(\sigma_{w}^{2}=0.3^{\circ^{2}}\right)$, denoted as $w^{i, j}$. In all, we have that

$$
y^{i, j}=\beta^{i, j}+f^{i, j}+w^{i, j}
$$

Table 1 summarizes the variables involved in the model of the collective pitch $\beta^{i, j}$ (depicted in Fig.3). The aim of this work is to develop an optimal, spatially and temporally integrated, model-based, closed-loop FE and FD strategy for the pitch misalignment of the WTs in the WF. As recalled in Fig.3, the collective pitch measurements $y^{i, j}\left(i=1, \ldots, n_{i}, j=1, \ldots, n_{j}\right)$, the dynamic power reference $P_{r}$ and the WS measurement at the wind mast $\hat{v}^{0}$, are available for FE and FD.

\footnotetext{
${ }^{1}$ The WF power demand is determined in different modes such as delta, absolute and frequency regulation modes [30]

${ }^{2}$ See $[31,32]$ for examples of WF static power reference computation algorithms.
} 


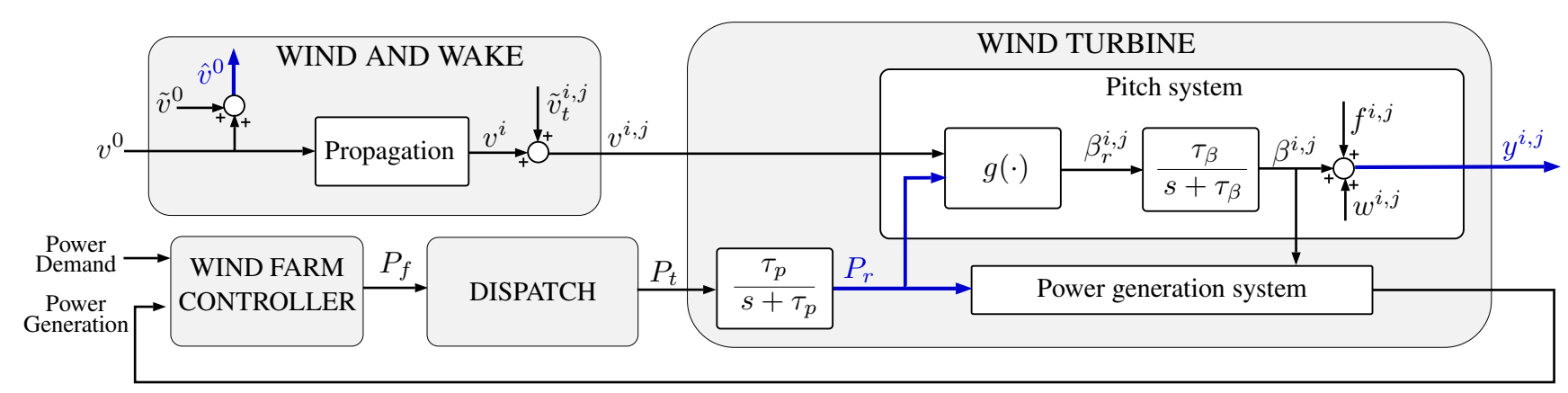

Figure 3. Structure of the WF benchmark. The signals which are available for FE and FD are depicted in blue.

\section{Pitch Misalignment Modeling}

It is well known that most continuous-time control systems are implemented digitally [33]. Thus, in this work, we work on the discrete-time framework and we first develop the discrete state-space model of the pitch system (2)-(4):

$$
\begin{aligned}
\beta_{k+1}^{i, j} & =\alpha \beta_{k}^{i, j}+(1-\alpha) g\left(\nu_{k}^{i, j}, P_{k}^{r}\right) \\
y_{k}^{i, j} & =\beta_{k}^{i, j}+f_{k}^{i, j}+w_{k}^{i, j}
\end{aligned}
$$

The discrete transfer function coefficient $\alpha$ is derived from $\tau_{\beta}$ in (2) with an appropriate sampling time, which we fix to $T_{s}=0.1 \mathrm{~s}$.

The FE and FD strategies developed in this work rely on augmented observers, which are widely utilized in the FE and FD literature (see [26,34] for detailed explanations on the advantages of these observers for FE and FD). Augmented observers are based on augmented models including both the system dynamics and the fault dynamics. In practice, the faut dynamics is not a priori known. Hence, the following assumption on the fault dynamics is introduced.

Assumption 1. The variation $\delta^{i, j}$ of the fault $f^{i, j}$ (i.e., $\left.\delta^{i, j}(k)=f^{i, j}(k+1)-f^{i, j}(k)\right)$ is energy bounded.

The faults verifying Assumption 1 can be modeled through

$$
\begin{aligned}
\xi_{k+1}^{i, j} & =a_{F} \xi_{k}^{i, j}+b_{F} \delta_{k}^{i, j} \\
f_{k}^{i, j} & =c_{F} \xi_{k}^{i, j}
\end{aligned}
$$

with $\xi^{i, j}$ being the fault state and $a_{F}=b_{F}=c_{F}=1$.

Remark 2. Assumption 1 is fairly general because it considers faults whose variations are slow with respect to the dynamics of the system. It covers thus the most typical faults in engineering systems such as abrupt and incipient faults [33,35]. In any case, the strategies developed in this work can be easily extended to systems whose faults do not verify Assumption 1. If more complex fault signals affected the system, model (6) should be modify accordingly. See, for instance, the models developed in $[34,36]$ for faults in the form of a polynomial or a sinusoid of the time.

The model (5) is augmented to include the fault dynamics (6) as

$$
\begin{aligned}
z_{k+1}^{i, j} & =\left[\begin{array}{ll}
\alpha & 0 \\
0 & 1
\end{array}\right] z_{k}^{i, j}+(1-\alpha) g\left(\nu_{k}^{i, j}, P_{k}^{r}\right)+\left[\begin{array}{l}
0 \\
1
\end{array}\right] \delta_{k}^{i, j} \\
y_{k}^{i, j} & =\left[\begin{array}{ll}
1 & 1
\end{array}\right] z_{k}^{i, j}+w_{k}^{i, j}
\end{aligned}
$$




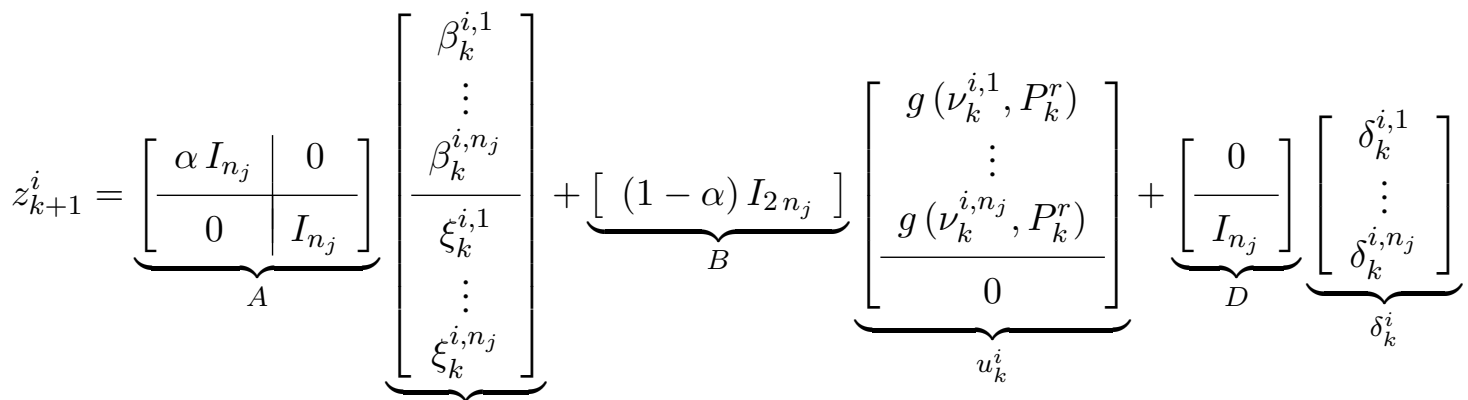

$$
\begin{aligned}
& \underbrace{\left[\begin{array}{c}
y_{k}^{i, 1} \\
\vdots \\
y_{k}^{i, n_{j}}
\end{array}\right]}_{y_{k}^{i}}=\underbrace{\left[\begin{array}{ll}
I_{n_{j}} & I_{n_{j}}
\end{array}\right]}_{C} z_{k}^{i}+\underbrace{\left[\begin{array}{c}
w_{k}^{i, 1} \\
\vdots \\
w_{k}^{i, n_{j}}
\end{array}\right]}_{w_{k}^{i}} \\
& \underbrace{\left[\begin{array}{c}
f_{k}^{i, 1} \\
\vdots \\
f_{k}^{i, n_{j}}
\end{array}\right]}_{f_{k}^{i}}=\underbrace{\left[\begin{array}{ll}
0 & I_{n_{j}}
\end{array}\right]}_{R} z_{k}^{i}
\end{aligned}
$$

where $z^{i, j}=\left[\begin{array}{ll}\beta^{i, j} & \xi^{i, j}\end{array}\right]^{T} \in \mathbb{R}^{2}$ is the extended state vector the collective pitch $\beta^{i, j}$ and the fault state $\xi^{i, j}$.

As we desire to obtain a spatially and temporally integrated FE and FD approach, we merge the temporal model (7) of all the WTs operating under similar wind conditions (i.e., all the WTs in the same row $i$ ). The discrete extended state-space model of the pitch systems of the $i$-th row of WTs can be written as detailed in (8) with $z^{i} \in \mathbb{R}^{2 n_{j}}$ being the extended state vector that stacks the collective pitch angles $\beta^{i, j}$ and the fault states $\xi^{i, j}$ of the WTs in the $i$-th row from $j=1$ to $j=n_{j}$. Similar applies to $u^{i} \in \mathbb{R}^{n_{j}}, y^{i} \in \mathbb{R}^{n_{j}}, w^{i} \in \mathbb{R}^{n_{j}}, f^{i} \in \mathbb{R}^{n_{j}}$ and $\delta^{i} \in \mathbb{R}^{n_{j}}$, which are the input, output, noise, fault and fault generator vectors.

The model (8) merges the temporal and spatial information regarding the pitch system of the WTs in the $i$-th row, which operate under similar wind conditions ${ }^{3}$. For ease of readability, let us omit hereafter the dependence of the system vectors on the number of row $i$ (e.g. $z_{k}$ stands for $z_{k}^{i}$ ):

$$
\begin{aligned}
z_{k+1} & =A z_{k}+B u_{k}+D \delta_{k}, \\
y_{k} & =C z_{k}+w_{k} \\
f_{k} & =R z_{k}
\end{aligned}
$$

Any individual fault $f[j]=f^{i, j}$ is extracted from the fault vector $f$ as $f[j]=F^{j} f$ where $F^{j}$ is the selection matrix defined as $F^{j}=\left[\begin{array}{lll}\mathbf{0}_{j-1} & 1 & \mathbf{0}_{n_{j}-j}\end{array}\right]$.

In the following, we utilize the model (9) to provide a FE and FD strategy of the pitch misalignments occurring in the $i$-th row of WTs in the $\mathrm{WF}^{4}$. We propose the following methodology:

1. To obtain open-loop estimates of the inputs (i.e., the pitch references).

2. To obtain and design a closed-loop model-based augmented FE observer that utilizes the previous open-loop estimates of the inputs.

3. To obtain and design statistical-based FD decision mechanisms with adaptive thresholds that process the previous closed-loop fault estimates.

\footnotetext{
${ }^{3}$ All the pitch systems of the WF are modelled through (8) with $i=1, \ldots, n_{i}$.

${ }^{4}$ All the pitch misalignments can be estimated and diagnosed with the proposed approach applied for $i=1, \ldots, n_{i}$
} 


\section{Wind Speed and Pitch Reference Estimation}

The first step to estimate the pitch misalignment faults is to provide an open-loop estimation strategy of the pitch references which depend on the unknown effective WSs. In this section, we first propose a propagation strategy to estimate the effective WSs and we bound the errors associated to the estimates. Then, we use the estimated effective WSs to estimate the pitch references and we bound the resulting pitch reference open-loop estimation errors. See the open-loop estimation box in Fig.5.

\subsection{Wind Speed Estimation}

We can propagate the WS measurement $\hat{\nu}^{0}$ to certain row $i$ in order to estimate the mean WS acting on the WTs in this row through different propagation models available in the literature (see [37] and the references therein). Based on the wind and wake model of the WF benchmark in [17], we propose to compute the estimated mean WS, named after $\hat{\nu}^{i}$, using the following wind propagation model:

$$
\hat{\nu}^{i}(t)=\frac{1}{K_{i}} \hat{\nu}^{i-1}\left(t-t_{p}^{i}(t)\right)
$$

where $K_{i}$ is a wake factor and satisfies $K_{i}=1$ for $i=1$ and $K_{i}=0.9$ for $i=2, \ldots, n_{i}-1$. The term $t_{p}^{i}(t)$ represents the propagation time between two consecutive rows and we propose to compute it as

$$
t_{p}^{i}(t)=\frac{l_{i}}{\hat{\nu}^{0}(t) /\left(K_{i}\right)^{i-1}}
$$

Then, the mean WS fulfils

$$
\nu^{i}=\hat{\nu}^{i}+\tilde{\nu}_{p}^{i}
$$

where $\tilde{\nu}_{p}^{i}$ is the propagation error. This error derives from both the use of the noisy measurement $\hat{\nu}^{0}$ and the propagation model mismatch. Fig.A.1 in Appendix A shows the WS estimation results for the $0^{\circ}$ layout.

Remark 3. In order to avoid sudden large propagation errors, the estimated mean WS is filtered using a low pass filter with the transfer function $H_{p}(s)=\frac{1}{s+1}$. Fig.A.2 in Appendix A shows that filtering the prorogated mean WS leads to smoother errors in the same order of magnitude as the errors of the non-filtered estimated WS.

According to (1) and (12), we deduce that the effective WS $\nu^{i, j}$ satisfies

$$
\nu^{i, j}=\hat{\nu}^{i}+\tilde{\nu}^{i, j}
$$

where $\tilde{\nu}^{i, j}$ is the total WS estimation error verifying

$$
\tilde{\nu}^{i, j}=\tilde{\nu}_{p}^{i}+\tilde{\nu}_{t}^{i, j}
$$

Note that the propagation error $\tilde{\nu}_{p}^{i}$ is common for all the WTs in the $i$-th row while the turbulence $\tilde{\nu}_{t}^{i, j}$ is different for each WT $(i, j)$. The following assumption on these errors is made.

Assumption 2. The errors in (14) can be considered to be bounded as follows.

- The propagation error $\tilde{\nu}_{p}^{i}$ of the WTs in the $i$-th can be considered to be bounded as $\left|\tilde{\nu}_{p}^{i}\right| \leq \lambda_{p}^{i}$, where $\lambda_{p}^{i}$ is different for each row of WTs $i$ (the propagation error increases with the distance between to the wind mast).

- The turbulence $\tilde{\nu}_{t}^{i, j}$ of the WT $(i, j)$ can be considered to be bounded as $\left|\tilde{\nu}_{t}^{i, j}\right| \leq \lambda_{t}$. We compute $\lambda_{t}$ as $\lambda_{t}=3 \sigma_{t}$.

In Appendix A we compute and validate these bounds through numerical simulations. Let us remark that this procedure is usually employed in observer-based applications $[38,39]$. 
Remark 4. The FE and FD strategies developed in this paper are independent of the wind propagation scheme. If other propagation strategies were used, the bounds $\lambda_{p}^{i}\left(i=1, \ldots, n_{i}\right)$ would vary accordingly. Similar applies to the turbulence component that, if considered to be differently distributed, it would be bounded with a different value $\lambda_{t}$.

\subsection{Pitch Reference Estimation}

The estimate $\hat{\nu}^{i}$ of the effective WS $\nu^{i, j}$ given by (10) is used to estimate the pitch reference (2) of the WTs in the $i$-th row as

$$
\hat{\beta}_{r}^{i, j}=g\left(\hat{\nu}^{i}, P^{r}\right)
$$

From (14), we deduce that the pitch reference estimation error can be disaggregated as

$$
g\left(\nu^{i, j}, P^{r}\right)-g\left(\hat{\nu}^{i}, P^{r}\right)=\underbrace{g\left(\nu^{i, j}, P^{r}\right)-g\left(\nu^{i}, P^{r}\right)}_{\tilde{u}^{i, j}}+\underbrace{g\left(\nu^{i}, P^{r}\right)-g\left(\hat{\nu}^{i}, P^{r}\right)}_{\tilde{u}^{i}}
$$

where $\tilde{u}^{i}$ derives from the propagation error $\tilde{\nu}_{p}^{i}$ (i.e., $\tilde{\nu}_{p}^{i}=\nu^{i}-\hat{\nu}^{i}$ ) and $\tilde{u}^{i, j}$ derives from the turbulence $\tilde{\nu}_{t}^{i, j}$ (i.e., $\left.\tilde{\nu}_{t}^{i, j}=\nu^{i, j}-\nu^{i}\right)$. Thus, the error $\tilde{u}^{i}$ is common for all the WTs in the $i$-th row while $\tilde{u}^{i, j}$ is different for each WT. It s clear from (16) that these errors depend on the WT operation conditions (i.e., they are different for the different values of $\hat{\nu}^{i}$ and $P^{r}$ ). For certain value of the estimated WS and of the dynamic power reference and for certain row $i$, one can compute the bounds of these errors with the values $\lambda_{p}^{i}$ and $\lambda_{t}$ (which are the bounds of the WS estimation errors in Assumption 2) as ${ }^{5}$

$$
\begin{aligned}
&\left|\tilde{u}^{i}\right| \leq \max \left\{\begin{array}{l}
\left|g\left(\hat{\nu}^{i}+\lambda_{p}^{i}, P^{r}\right)-g\left(\hat{\nu}^{i}, P^{r}\right)\right|, \\
\left|g\left(\hat{\nu}^{i}-\lambda_{p}^{i}, P^{r}\right)-g\left(\hat{\nu}^{i}, P^{r}\right)\right|
\end{array}\right\} \\
&\left|\tilde{u}^{i, j}\right| \leq \max \left\{\begin{array}{l}
\left|g\left(\hat{\nu}^{i}+\lambda_{p}^{i}+\lambda_{t}, P^{r}\right)-g\left(\hat{\nu}^{i}+\lambda_{p}^{i}, P^{r}\right)\right|, \\
\left|g\left(\hat{\nu}^{i}+\lambda_{p}^{i}-\lambda_{t}, P^{r}\right)-g\left(\hat{\nu}^{i}+\lambda_{p}^{i}, P^{r}\right)\right|, \\
\left|g\left(\hat{\nu}^{i}-\lambda_{p}^{i}+\lambda_{t}, P^{r}\right)-g\left(\hat{\nu}^{i}-\lambda_{p}^{i}, P^{r}\right)\right|, \\
\left|g\left(\hat{\nu}^{i}-\lambda_{p}^{i}-\lambda_{t}, P^{r}\right)-g\left(\hat{\nu}^{i}-\lambda_{p}^{i}, P^{r}\right)\right|
\end{array}\right\}
\end{aligned}
$$

Designing an augmented observer that only considers the biggest possible value of these bounds and omits their differences among the WT operation range would be too conservative. On the other hand, designing an augmented observer considering all the values of these errors among the WT operation range would be too computationally costly. For this reason, we consider an intermediate solution and we propose to design an augmented observer considering the biggest value of these errors on different subsets of the WT operation range. This option leads to a switched augmented observer that prevents the fault estimates to be affected by the errors in each subset. Hence, we bound the errors $\tilde{u}^{i}$ and $\tilde{u}^{i, j}$ as follows.

The variables $\hat{\nu}^{i}$ and $P^{r}$, which define the WT operation conditions, can be considered to be bounded by the sets

$$
\begin{aligned}
\hat{\nu}^{i} & \in \Omega_{\nu}, \Omega_{\nu}:=\left\{\underline{\nu}<\hat{\nu}^{i}<\bar{\nu}\right\} \\
P^{r} \in \Omega_{p}, & \Omega_{p}:=\left\{\underline{P}<P^{r}<\bar{P}\right\}
\end{aligned}
$$

where $\underline{\nu}, \bar{\nu}, \underline{P}$ and $\bar{P}$ are the minimum and maximum possible values of these parameters (which we fix to $\underline{\nu}=0$ $\mathrm{m} / \mathrm{s}, \bar{\nu}=25 \mathrm{~m} / \mathrm{s}, \underline{P}=0 \mathrm{MW}$ and $\bar{P}=4.8 \mathrm{MW}$ ). The parameter vector $\theta=\left[\begin{array}{ll}\hat{\nu}^{i} & P^{r}\end{array}\right]^{T}$ lies then in $\Theta=\Omega_{\nu} \times \Omega_{p}$. Let us partition the parameter set $\Theta$ into $N_{\theta}$ subsets $\left\{\Theta^{(q)}\right\}_{q \in\left\{1, \ldots, N_{\theta}\right\}}$ by dividing $\Omega_{\nu}$ into $N_{\nu}$ intervals (i.e., $\left\{\Omega_{\nu}^{\left(q_{\nu}\right)}\right\}_{q_{\nu} \in\left\{1, \ldots, N_{\nu}\right\}}$ ) and $\Omega_{p}$ into $N_{p}$ intervals (i.e., $\left\{\Omega_{p}^{\left(q_{p}\right)}\right\}_{q_{p} \in\left\{1, \ldots, N_{p}\right\}}$ ). Thus, we have $N_{\theta}=N_{\nu} \cdot N_{p}$ (see Fig.4).

\footnotetext{
${ }^{5}$ Note that we express these bounds in terms of $\hat{\nu}^{i}$ because the variables $\nu^{i, j}$ and $\nu^{i}$ are not available.
} 


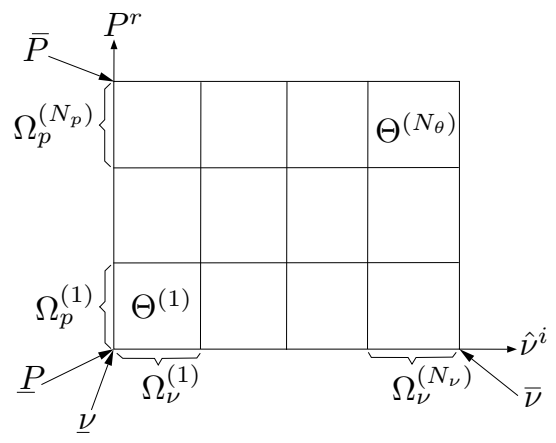

Figure 4. Partition of the two-dimensional parameter set $\left(N_{\nu}=4\right.$ and $\left.N_{p}=3\right)$.

For each row $i, \tilde{u}^{i}$ and $\tilde{u}^{i, j}$ are bounded as

$$
\begin{array}{cl}
\left|\tilde{u}^{i}\right| \leq \lambda_{1, q}^{i} & \text { if } \theta \in \Theta^{(q)} \\
\left|\tilde{u}^{i, j}\right| \leq \lambda_{2, q}^{i} & \text { if } \theta \in \Theta^{(q)}
\end{array}
$$

where

$$
\lambda_{1, q}^{i}=\max _{\theta \in \Theta^{(q)}}\left\{\begin{array}{l}
\left|g\left(\hat{\nu}^{i}, P^{r}\right)-g\left(\hat{\nu}^{i}+\lambda_{p}^{i}, P^{r}\right)\right| \\
\left|g\left(\hat{\nu}^{i}, P^{r}\right)-g\left(\hat{\nu}^{i}-\lambda_{p}^{i}, P^{r}\right)\right|
\end{array}\right\}
$$

Similar applies to $\lambda_{2, q}^{i}$, whose value is deduced from (17b) along the values $\theta$ such that $\theta \in \Theta^{(q)}$. In Appendix A, we detail the computation of these bounds.

\section{Pitch Misalignment Estimation}

In this section, we develop a FE strategy based on a switched augmented observer. The augmented observer is based on the augmented model (9) and utilizes the open-loop estimates of the pitch references obtained in Section 4. We propose a multiobjective optimal design strategy of the augmented observer that allows an optimal adjustment to the different levels of uncertainties encountered along the WT operation range.

\subsection{Estimator Architecture}

The pitch references $g\left(\nu^{i, j}, P^{r}\right)$ are not available. Then, we build a model-based observer for the system (9) based on the open-loop estimates (15). Provided that the bounds of the errors associated to these estimated variables depend on $\theta$, we propose to use parameter-dependent gain matrices in the observer. Then, we have

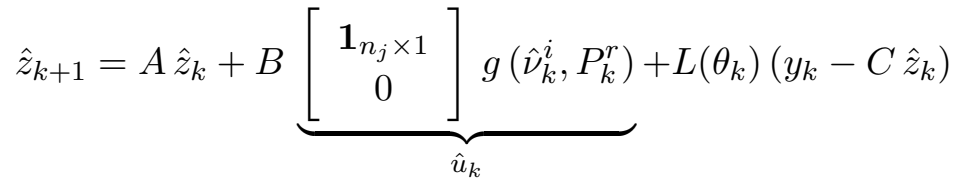

$$
\begin{aligned}
& \hat{f}_{k}=R \hat{z}_{k}+K\left(\theta_{k}\right)\left(y_{k}-C \hat{z}_{k}\right)
\end{aligned}
$$

where $L\left(\theta_{k}\right)$ and $K\left(\theta_{k}\right)$ are the parameter-dependent design gain matrices of appropriate dimensions. Given the bounds in (19), we define $L\left(\theta_{k}\right)$ and $K\left(\theta_{k}\right)$ as

$$
\begin{gathered}
L\left(\theta_{k}\right)=L_{q} \quad \text { if } \theta_{k} \in \Theta^{(q)} \\
K\left(\theta_{k}\right)=K_{q} \quad \text { if } \theta_{k} \in \Theta^{(q)}
\end{gathered}
$$


and the design gain matrices become the finite set of gain matrices $L_{q}$ and $K_{q}$ with $q=1, \ldots, N_{\theta}$ (i.e., $L\left(\theta_{k}\right) \in$ $\left\{L_{1}, \ldots, L_{q}, \ldots, L_{N_{\theta}}\right\}$ and $K\left(\theta_{k}\right) \in\left\{K_{1}, \ldots, K_{q}, \ldots, K_{N_{\theta}}\right\}$ ). In the following, we present a multiobjective approach to offline design these matrices. To this aim, define the extended state estimation error as $\tilde{z}_{k}=z_{k}-\hat{z}_{k}$ and the fault estimation error as $\tilde{f}_{k}=f_{k}-\hat{f}_{k}$. It follows that

$$
\begin{aligned}
\tilde{z}_{k+1} & =\left(A-L\left(\theta_{k}\right) C\right) \tilde{z}_{k}+B\left(u_{k}-\hat{u}_{k}\right)+D \delta_{k}-L\left(\theta_{k}\right) w_{k} \\
\tilde{f}_{k} & =\left(R-K\left(\theta_{k}\right) C\right) \tilde{z}_{k}-K\left(\theta_{k}\right) w_{k}
\end{aligned}
$$

The difference $u_{k}-\hat{u}_{k}$ refers to the pitch reference estimation errors. As detailed in Section 4.2, this error can be disaggregated as

$$
u_{k}-\hat{u}_{k}=\left[\begin{array}{c}
g\left(\nu_{k}^{i, 1}, P_{k}^{r}\right)-g\left(\hat{\nu}_{k}^{i}, P_{k}^{r}\right) \\
\vdots \\
g\left(\nu_{k}^{i, n_{j}}, P_{k}^{r}\right)-g\left(\hat{\nu}_{k}^{i}, P_{k}^{r}\right) \\
0
\end{array}\right]=\underbrace{\left[\begin{array}{c}
\mathbf{1}_{n_{j} \times 1} \\
0
\end{array}\right]}_{E_{0}} \underbrace{\tilde{u}_{k}^{i}}_{p_{k}}+\underbrace{\left[\begin{array}{c}
I_{n_{j}} \\
0
\end{array}\right]}_{F_{0}} \underbrace{\left[\begin{array}{c}
\tilde{u}_{k}^{i, 1} \\
\vdots \\
\tilde{u}_{k}^{i, n_{j}}
\end{array}\right]}_{t_{k}}
$$

where $p_{k}$ is the pitch reference estimation error due to the error on the WS propagation scheme and it is common to all the WTs in the $i$-th row. Vector $t_{k}$ contains the pitch reference estimation error due to the WS estimation error caused by the turbulence of each WT. Provided the bounds (19), we deduce that the vectors $p_{k}$ and $t_{k}$ are bounded as

$$
\begin{array}{cl}
\left\|p_{k}\right\|_{\infty} \leq \lambda_{1, q}^{i} & \text { if } \theta_{k} \in \Theta^{(q)} \\
\left\|t_{k}\right\|_{\infty} \leq \lambda_{2, q}^{i} & \text { if } \quad \theta_{k} \in \Theta^{(q)}
\end{array}
$$

Remark 5. Since $p_{k}$ is common to all the WTs in the $i$-th row, the effect of the wind propagation error on FE is diminished when all these WTs are considered simultaneously by the same observer. This fact shows the advantage of building the temporally and spatially integrated fault estimator (21) based on the model (9), which merges all the information of the WTs in the $i$-th row.

\subsection{Estimator Design}

From (23)-(24), it yields

$$
\begin{aligned}
\tilde{z}_{k+1} & =\left(A-L\left(\theta_{k}\right) C\right) \tilde{z}_{k}+E p_{k}+F t_{k}+D \delta_{k}-L\left(\theta_{k}\right) w_{k} \\
\tilde{f}_{k} & =\left(R-K\left(\theta_{k}\right) C\right) \tilde{z}_{k}-K\left(\theta_{k}\right) w_{k}
\end{aligned}
$$

with $E=B E_{0}$ and $F=B F_{0}$. The fault estimation error depends on the following exogenous signals:

(a) the fault generator $\delta$ satisfying Assumption 1,

(b) the noises $w$ satisfying $w \sim \mathcal{N}(0, W)$ with $W=\sigma_{w}^{2} I_{n_{j}}$,

(c) the unknown inputs $p$ and $t$ satisfying the bounds (25).

The error caused by $\delta$ describes the fault tracking ability of the observer and the errors caused by $w, p$ and $t$ describe the robustness of the observer against noises and uncertainties. Hence, we propose to characterize the fault tracking ability of the observer by the cumulative squared error (CSE) experienced by each estimate $\hat{f}[j]$ (i.e., 
$\|\tilde{f}[j]\|_{2}^{2}=\sum_{k=1}^{\infty} \tilde{f}[j]^{2}$ ) when it faces a unitary impulse $\delta[m]$ (which generates a unitary step fault $f[m]$ ):

$$
\begin{array}{cc}
\gamma_{m}^{j}=\max _{\theta \in \Theta} \frac{\|\tilde{f}[j]\|_{2}^{2}}{\|\delta[m]\|_{2}^{2}}, & \delta[n]=\{1,0,0, \ldots\}, \forall n \neq m, \\
& w=0, p=0, t=0
\end{array}
$$

Similarly, we characterize the robustness of the observer by the root-mean-square (RMS) error experienced by estimate $\hat{f}[j]$ (i.e., $\|\tilde{f}[j]\|_{\mathrm{RMS}}=\lim _{K \rightarrow \infty} \sqrt{\frac{1}{K} \sum_{k=1}^{K} \tilde{f}[j]^{2}}$ ) due to the noises (b) and the UIs (c):

$$
\begin{array}{ll}
\gamma_{w}^{j}=\max _{\theta \in \Theta} \frac{\|\tilde{f}[j]\|_{\mathrm{RMS}}^{2}}{\sigma_{w}^{2}}, & \delta=0, p=0, t=0 \\
\gamma_{p}^{j}=\max _{\theta \in \Theta} \frac{\|\tilde{f}[j]\|_{\mathrm{RMS}}^{2}}{\|p\|_{\infty}^{2}}, & \delta=0, w=0, t=0 \\
\gamma_{t}^{j}=\max _{\theta \in \Theta} \frac{\|\tilde{f}[j]\|_{\mathrm{RMS}}^{2}}{\|t\|_{\infty}^{2}}, & \delta=0, w=0, p=0
\end{array}
$$

There exists a trade-off between ameliorating the fault tracking ability of the observer and its robustness [40,41]. From the practical viewpoint, it is of considerable interest to achieve certain tracking ability and to minimize the effect of the noises and uncertainties on the estimations [42]. Thus, we propose to design the observer gain matrices through the following optimization problem

$$
\begin{array}{cl}
\underset{\left\{L_{q}, K_{q}\right\}_{q \in\left\{1, \ldots, n_{\theta}\right\}}}{\operatorname{minimize}} & \sum_{j=1}^{n_{j}}\left(\gamma_{w}^{j} \sigma_{w}^{2}+\gamma_{p}^{j}\|p\|_{\infty}^{2}+\gamma_{t}^{j}\|t\|_{\infty}^{2}\right) \\
\text { subject to } & \gamma_{m}^{j} \leq \bar{\gamma}_{m}^{j}, \quad \forall j, m \\
& \text { stability of }(26), \quad \theta \in \Theta
\end{array}
$$

Remark 6. Note that $\gamma_{m}^{j}$ with $m \neq j$ denotes the CSE experienced by the fault estimate in the $j$-th fault channel due to a unitary step fault in another channel $m$ while $\gamma_{j}^{j}$ denotes the CSE experienced due to a unitary step fault in the own channel $j$. Hence, the CSE requirements $\bar{\gamma}_{m}^{j}\left(m=1, \ldots, j-1, j+1, \ldots, n_{j}\right)$ are generally chosen to be smaller than the corresponding CSE requirement ${ }^{6} \bar{\gamma}_{j}^{j}$.

The system (26) operates in a finite set of multiple modes because

$$
\begin{aligned}
L\left(\theta_{k}\right) & \in\left\{L_{q}\right\}_{q \in\left\{1, \ldots, n_{\theta}\right\}} \\
K\left(\theta_{k}\right) & \in\left\{K_{q}\right\}_{q \in\left\{1, \ldots, n_{\theta}\right\}}
\end{aligned}
$$

A general theory of such systems is developed in the switched systems community [43-45]. The results for switched systems under arbitrary switchings are rather conservative and much tighter results can be developed if further assumptions hold [46]. Take as an example a Markovian jump linear system where the switching process can be described by a Markov chain [47]. In this work, we consider that the parameter $\theta$ is slow-varying compared to the dynamics of (26). Thus, the membership of $\theta$ to a subset $\Theta^{(q)}$ infrequently switches to another subset $\Theta^{(p \neq q)}$. In this fashion, we translate the optimization problem (28) into another design problem ensuring global stability in

\footnotetext{
${ }^{6}$ In order to approximately fix the $\chi \%$ settling times of the observer, we can approximate the fault observer responses to the response of a first-order system. Hence, if we fix $\bar{\gamma}_{j}^{j}=\frac{1}{1-\left(1+\log (1-\chi / 100) / \bar{T}^{j}\right)^{2}}$, the $\chi \%$ settling time of the $j$-th fault estimate due to a step fault $f[j]$ is approximated to $\bar{T}^{j}$.
} 
the set $\Theta$ and certain local steady-state performance in each subset $\Theta^{(q)}$ :

$$
\begin{array}{cl}
\underset{\left\{L_{q}, K_{q}\right\}_{q \in\left\{1, \ldots, n_{\theta}\right\}}}{\operatorname{minimize}} & \sum_{q=1}^{N_{\theta}} \sum_{j=1}^{n_{j}}\left(\gamma_{w, q}^{j} \sigma_{w}^{2}+\gamma_{p, q}^{j}\left(\lambda_{1, q}^{i}\right)^{2}+\gamma_{t, q}^{j}\left(\lambda_{2, q}^{i}\right)^{2}\right) \\
\text { subject to } & \gamma_{m, q}^{j} \leq \bar{\gamma}_{m}^{j}, \quad \forall j, m, q \\
& \text { stability of (26) }, \quad \theta \in \Theta
\end{array}
$$

where $\gamma_{m, q}^{j}, \gamma_{w, q}^{j}, \gamma_{p, q}^{j}, \gamma_{t, q}^{j}$ denote the maximums in (27) when $\theta \in \Theta^{(q)}$, e.g.,

$$
\gamma_{m, q}^{j}=\max _{\theta \in \Theta^{(q)}} \frac{\|\tilde{f}[j]\|_{2}^{2}}{\|\delta[m]\|_{2}^{2}}, \quad \begin{gathered}
\delta_{k}[m]=\{1,0,0, \ldots\} \\
\delta[n]=0, \forall n \neq m \\
w=0, p=0, t=0
\end{gathered}
$$

Unlike $\gamma_{m}^{j}, \gamma_{w}^{j}, \gamma_{p}^{j}, \gamma_{t}^{j}$, which depend on $\left\{L_{q}, K_{q}\right\}_{q \in\left\{1, \ldots, n_{\theta}\right\}}, \gamma_{m, q}^{j}, \gamma_{w, q}^{j}, \gamma_{p, q}^{j}, \gamma_{t, q}^{j}$ only depend on $L_{q}$ and $K_{q}$. Note that in (30), we have taken into account that $\|p\|_{\infty} \leq \lambda_{1, q}^{i}$ if $\theta \in \Theta^{(q)}$ because $\left\|p_{k}\right\|_{\infty}$ satisfies (25). Similar applies to $\|t\|_{\infty}$. In all, an observer designed through the optimization problem (30) is globally stable inside the set $\Theta$ regardless of the parameter dynamics and it guarantees certain local performance trade-off in each subset $\Theta^{(q)}$ provided infrequent changes of subset membership. Similar approaches are utilized in practical works as [48-50].

Remark 7. As introduced in Section 4.2, the density $N_{\theta}$ of the grid $\left\{\Theta^{(q)}\right\}$ is to be determined from a trade-off between having a few gridding intervals that ensure reduced computational burden and infrequent changes of subset membership but introduce conservatism, or a lot of gridding intervals causing heavy computational time and more frequent changes of subset membership but reducing performance conservatism. In this work, we choose $N_{\nu}=N_{p}=7$ (see Appendix A). This gridding is a posteriori validated through the numerical simulations in Section 9 .

Regarding the stability requirement in (30), it should be noted that even when all the subsystems of a switched system are stable, such a system may fail to preserve stability. It is well-known in switched systems theory that a necessary and sufficient condition to ensure the stability of (26) is the existence of $N_{\theta}$ positive-definite matrices $P_{1}, \ldots, P_{N_{\theta}}$ satisfying ([43])

$$
\left(A-L_{q} C\right)^{T} P_{p}\left(A-L_{q} C\right)-P_{q} \preceq 0, \quad \forall(q, p) \in\left\{1, \ldots N_{\theta}\right\} \times\left\{1, \ldots N_{\theta}\right\}
$$

Regarding the performance requirements in (30), let us apply the $\mathcal{Z}$ transform to (26) when $\theta \in \Theta^{(q)}$; it yields

$$
\tilde{f}[j](z)=\sum_{m=1}^{n_{j}}\left(G_{m, q}^{j}(z) \delta[m](z)\right)+G_{w, q}^{j}(z) w(z)+G_{p, q}^{j}(z) p(z)+G_{t, q}^{j}(z) t(z)
$$

with $G_{m, q}^{j}(z)=-M_{q}^{j}(z) D\left(F^{m}\right)^{T}, G_{w, q}^{j}(z)=M_{q}^{j}(z) L_{q}+K_{q}, G_{p, q}^{j}(z)=M_{q}^{j}(z) E, G_{t, q}^{j}(z)=M_{q}^{j}(z) F$, 295 and $M_{q}^{j}(z)=F^{j}\left(R-K_{q} C\right)\left(z I-A+L_{q} C\right)^{-1}$. It is well-known that $\gamma_{m, q}^{j}, \gamma_{w, q}^{j}, \gamma_{p, q}^{j}, \gamma_{t, q}^{j}$ fulfil [51]

$$
\begin{aligned}
\gamma_{m, q}^{j} & =\left\|G_{m, q}^{j}(z)\right\|_{2}^{2}, \\
\gamma_{w, q}^{j} & =\left\|G_{w, q}^{j}(z)\right\|_{2}^{2}, \\
\gamma_{p, q}^{j} & \leq\left\|G_{p, q}^{j}(z)\right\|_{\infty}^{2}, \\
\gamma_{t, q}^{j} & \leq\left\|G_{t, q}^{j}(z)\right\|_{\infty}^{2}
\end{aligned}
$$

where $\|G(z)\|_{2}$ and $\|G(z)\|_{\infty}$ denote, respectively, the $\mathscr{H}_{2}$ norm and the $\mathscr{H}_{\infty}$ norm of a system $G(z)$. In all, (30) 
can be rewritten as

$$
\begin{array}{cl}
\underset{\left\{L_{q}, K_{q}, P_{q}, Q_{q}\right\}_{q} \in\left\{1, \ldots, n_{\theta}\right\}}{\operatorname{minimize}} & \sum_{q=1}^{N_{\theta}} \sum_{j=1}^{n_{j}}\left(\left\|G_{w, q}^{j}(z)\right\|_{2}^{2} \sigma_{w}^{2}+\left\|G_{p, q}^{j}(z)\right\|_{\infty}^{2}\left(\lambda_{1, q}^{i}\right)^{2}+\left\|G_{t, q}^{j}(z)\right\|_{\infty}^{2}\left(\lambda_{2, q}^{i}\right)^{2}\right) \\
\text { subject to } & \left\|G_{m, q}^{j}(z)\right\|_{2}^{2} \leq \bar{\gamma}_{m}^{j}, \quad \forall j, m, q \\
& \text { matrix inequalities in (32) }
\end{array}
$$

This optimization problem ensures global stability in the set $\Theta$ and certain local steady-state performance in each subset $\Theta^{(q)}$. The problem (35) is nonlinear and it can be solved using different heuristic optimization methods such as genetic algorithms. In Appendix B, we briefly show how to introduce some slack variables into the problem (35) and translate it into a convex optimization problem based on linear matrix inequalities (LMIs) [52,53].

Remark 8. From (35), it is straightforward to deduce that the proposed optimization design problem is easily adaptable to different levels of measurement noise (represented by the sensor noise variance $\sigma_{w}^{2}$ ) and of pitch reference uncertainties (represented by the estimation error bounds $\lambda_{1, q}^{i}$ and $\lambda_{1, q}^{i}$ for $q=1, \ldots, n_{\theta}$ ).

\section{Pitch Misalignment Diagnosis}

In this section, we develop FD decision mechanisms that compare the closed-loop fault estimates provided by the observer (21) to thresholds. We propose to compute statistical-based adaptive thresholds through the False Alarm Rate criterion that allow achieving a high diagnosability performance.

\subsection{Diagnoser Architecture}

Regarding FD, we set the following set of decisions $\left(j=1, \ldots, n_{j}\right)$ :

$$
\begin{cases}\text { if }\left|\hat{f}_{k}[j]\right| \geq J_{k}[j] & \text { Fault } f[j] \\ \text { otherwise } & \text { No fault } f[j]\end{cases}
$$

where $J[j]$ is an adaptive (time-varying) threshold to be computed online. In the following, we show a statisticalbased procedure to online compute the adaptive thresholds $J[j]$ of the decision mechanism.

\subsection{Diagnoser Design}

To characterize the behaviour of the fault diagnoser (36), we propose to use the parameters defined as follows.

- The false alarm rate (FAR) of the fault $j$, which we denote as $\phi^{j}$, is the probability of rising alarms of the $j$-th fault when no fault exists (i.e., $f=0$ ).

- The minimum diagnosable fault $j$ (MDF), which we denote as $v^{j}$, is the smallest value of a constant fault $f[j]$ which ensures that the alarm of fault $j$ raises at some sample $k_{j}$ provided the non-existence of other faults and disturbances (i.e., $f[m \neq j]=0, w=0, p=0, t=0$ ).

- The diagnosis time (DT) of fault $j$, which we denote as $\tau^{j}$, is the time elapsed between the sample $k_{a}$ of appearance of a fault $f[j]$ and the sample $k_{j}$ in which the alarm is raised provided the non-existence of other faults and disturbances (i.e., $f[m \neq j]=0, w=0, p=0, t=0$ ).

The diagnosers (36) consist of both the thresholds $J[j]$ and the estimates $\hat{f}[j]$ provided by (21). The values of the thresholds $J[j]$ fix the trade-off between the certainness and uncertainness of the diagnosis decision. Then, for certain estimator (21), as the thresholds $J[j]$ increase, so does the certainness of the decisions and the FARs 


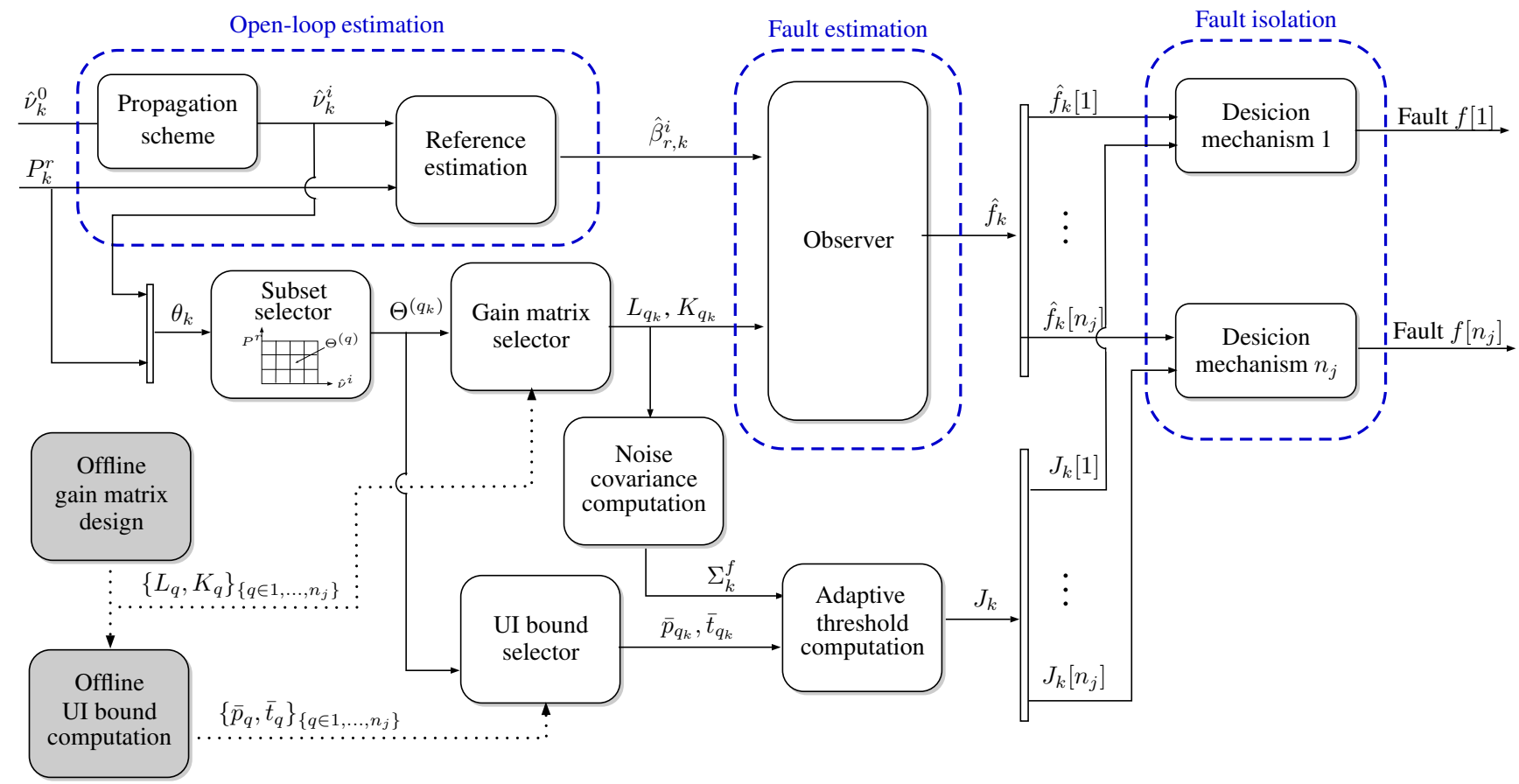

Figure 5. FE and FD strategy for each row of WTs $i$ ( $\square$ : Online computation, $\square$ : Offline computation).

decrease while the MDFs and DTs increase. Since the observer gain matrices are already designed through the optimization problem (35), we can only arbitrarily fix the thresholds $J[j]$. Hence, it is only possible to guarantee a performance requirement over one of the aforementioned diagnosis performance parameters. We propose to online compute the adaptive thresholds for guaranteeing certain FARs of the diagnoser.

The requirement over the FARs concerns the case in which $\delta=0$ and $f=0$ (i.e., $\hat{f}=-\tilde{f}$ ). In this case, the estimation error sources are the noises (b) and the UIs (c). In the following, we characterize the probability that, in the absence of faults, $\hat{f}[j]$ exceeds ceratin value due to each of these error sources. First, the signal $\hat{f}[j]$ due to the noises $w$ is zero-mean and Gaussian because the noises $w$ are zero-mean and Gaussian. Its time-varying variance is equal to the marginal variance of the fault estimation error $\tilde{f}[j]$, which is given by the $j$-th diagonal element of $\Sigma_{k}^{f}=\mathbb{E}\left\{\hat{f}_{k} \hat{f}_{k}^{T}\right\}$ (i.e., $\Sigma_{k}^{f}[j, j]$ ) and it can be computed as

$$
\begin{aligned}
\Sigma_{k+1} & =\left(A-L\left(\theta_{k}\right) C\right) \Sigma_{k}\left(A-L\left(\theta_{k}\right) C\right)^{T}+L\left(\theta_{k}\right) W L\left(\theta_{k}\right)^{T} \\
\Sigma_{k}^{f} & =\left(R-K\left(\theta_{k}\right) C\right) \Sigma_{k}\left(R-K\left(\theta_{k}\right) C\right)^{T}+K\left(\theta_{k}\right) W K\left(\theta_{k}\right)^{T}
\end{aligned}
$$

with $L\left(\theta_{k}\right)$ and $K\left(\theta_{k}\right)$ being selected through $(22)^{7}$. Taking account of the properties of the probability distribution function of a zero-mean Gaussian variable, if $\delta=0, p=0$ and $t=0$, we have that

$$
\mathbb{P}\left\{\left|\hat{f}_{k}[j]\right|>\Phi_{Z}^{-1}\left(1-\phi^{j} / 2\right) \sqrt{\Sigma_{k}^{f}[j, j]}\right\} \leq \phi^{j}
$$

with $\Phi_{Z}^{-1}(\cdot)$ being the inverse cumulative distribution function of a standard normal variable.

Second, the statistical properties of the signal $\hat{f}[j]$ due to the UIs $p$ and $t$ are unknown. Thus, we propose to approximate its probability distribution function to a zero-mean uniform distribution. If $\theta \in \Theta^{(q)}, \delta=0, w=0$ and $t=0$, the inequality

$$
\|\tilde{f}[j]\|_{\mathrm{RMS}} \leq\left\|G_{p, q}^{j}(z)\right\|_{\infty} \lambda_{1, q}^{i}
$$

\footnotetext{
${ }^{7}$ These Riccati equations have been obtained with $\delta(z)=0, p(z)=0, t(z)=0$ and using an internal realization of the transfer function between $w$ and $\tilde{f}$.
} 
holds for infrequent changes of subset membership ${ }^{8}$. Thus, taking account of the properties of the probability distribution function of a zero-mean uninform variable ${ }^{9}$, we have that

$$
\mathbb{P}\left\{\left|\hat{f}_{k}[j]\right|>\sqrt{3}\left\|G_{p, q}^{j}(z)\right\|_{\infty} \lambda_{1, q}^{i}\right\} \approx 0
$$

Similar applies to the error caused by $t$.

In all, given these probabilities, we online compute the adaptive thresholds $J[j]$ for approximately bounding the FARs (i.e., $\phi^{j} \lesssim \bar{\phi}^{j}$ for $j=1, \ldots, n_{j}$ ) as

$$
J_{k}[j]=\Phi_{Z}^{-1}\left(1-\bar{\phi}^{j} / 2\right) \sqrt{\Sigma_{k}^{f}[j, j]}+\bar{p}\left(\theta_{k}\right)+\bar{t}\left(\theta_{k}\right), \quad \forall j
$$

with

$$
\begin{aligned}
& \bar{p}\left(\theta_{k}\right)=\bar{p}_{q} \quad \text { if } \theta_{k} \in \Theta^{(q)} \\
& \bar{t}\left(\theta_{k}\right)=\bar{t}_{q} \quad \text { if } \theta_{k} \in \Theta^{(q)}
\end{aligned}
$$

and where $\bar{p}_{q}$ and $\bar{t}_{q}$ are computed offline as

$$
\begin{aligned}
\bar{p}_{q} & =\sqrt{3}\left\|G_{p, q}^{j}(z)\right\|_{\infty} \lambda_{1, q}^{i} \\
\bar{t}_{q} & =\sqrt{3}\left\|G_{t, q}^{j}(z)\right\|_{\infty} \lambda_{2, q}^{i}
\end{aligned}
$$

with the designed gains $L_{q}$ and $K_{q}$ for $q=1, \ldots, N_{\theta}$. In all, the proposed FE and FD strategy is depicted in Fig.5.

Remark 9. In order to avoid false alarms when the membership of $\theta$ to a subset $\Theta^{(q)}$ changes to a subset $\Theta^{(p \neq q)}$ such that $\left(\bar{p}_{q}+\bar{t}_{q}\right)>\left(\bar{p}_{p}+\bar{t}_{p}\right)$ we use the refined adaptive threshold $J_{k}^{*}[j]$ defined as

$$
J_{k}^{*}[j]=\left\{\begin{array}{cl}
\alpha_{j} J_{k-1}^{*}[j]+\left(1-\alpha_{j}\right) J_{k}[j] & \text { if } J_{k}[j]<J_{k-1}^{*}[j] \\
J_{k}[j] & \text { otherwise }
\end{array}\right.
$$

with $\alpha_{j}$ defining a sufficiently slow filter which can be validated through numerical simulations (e.g., $\left.\alpha_{j}=0.90\right)$.

Remark 10. The propagation error $p$ is a high-frequency signal and we can model its dynamics as $p(z)=$ $\frac{z-1}{z-\alpha_{f}} \eta(z)$ where $\eta$ is a random variable verifying $\|\eta\|_{\infty}=\|p\|_{\infty}$ and $\alpha_{f} \lesssim 1$ (we choose $\alpha_{f}=0.99$ ). Thus, we replace $\left\|G_{p, q}^{j}(z)\right\|_{\infty}$ by $\left\|G_{p, q}^{j}(z) \frac{z-1}{z-\alpha_{f}}\right\|_{\infty}$ in (43a). Similar applies to $t$; hence, we replace $\left\|G_{t, q}^{j}(z)\right\|_{\infty}$ by $\left\|G_{t, q}^{j}(z) \frac{z-1}{z-\alpha_{f}}\right\|_{\infty}$ in (43b). With this, we give more weight to high-frequency components w.r.t. low-frequency components that would lead to a conservative computation of the thresholds of the decision mechanisms.

\section{Improvement of Performance with Fault Simultaneity Restrictions}

The dependence of the thresholds on the variances of the noises and on the peak bounds of the UIs (see (41)) may compromise the performance of the diagnoser. Even if the effect of the noises and UIs is attenuated (see the optimization problem (35)), large values of the variances of the noises and, especially, of the bounds of the UIs may lead to large values of the MDFs and DTs. In such a case, it would not be possible to diagnose through (36) the occurrence of small faults to which the system may be prone. Thus, we propose to improve the performance of the diagnoser at the cost of a constraint regarding the simultaneity of faults.

To do so, we build a bank of $n_{j}$ observers $b\left(b=1, \ldots, n_{j}\right)$. Each observer $b$ aims to estimate the auxiliary fault

\footnotetext{
${ }^{8}$ From the definition of $\gamma_{p, q}^{j}$, it yields $\|\tilde{f}[j]\|_{\mathrm{RMS}} \leq \gamma_{p, q}^{j}\|p\|_{\infty}$; from (34), $\gamma_{p, q}^{j} \leq\left\|G_{p, q}^{j}(z)\right\|_{\infty}$; and from (25), $\|p\|_{\infty} \leq \lambda_{1, q}^{i}$.

${ }^{9}$ If $x$ is a zero-mean uninform variable, then $|x|<\sqrt{3}\|x\|_{\text {RMS. }}$.
} 
vector

$$
\bar{f}_{k}^{b}=\left[\begin{array}{llllll}
f_{k}^{i, 1} & \ldots & f_{k}^{i, b-1} & f_{k}^{i, b+1} & \ldots & f_{k}^{i, n_{j}}
\end{array}\right]^{T}
$$

The discrete state-space model of the pitch systems of the $i$-th row of WTs including the dynamics of $\bar{f}^{b}$ can be written as

$$
\begin{aligned}
\bar{z}_{k+1}^{b} & =\bar{A} \bar{z}_{k}^{b}+\bar{B} u_{k}+\bar{D} \bar{\delta}_{k}^{b} \\
y_{k} & =\bar{C} \bar{z}_{k}^{b}+w_{k}+\bar{G}^{b} f_{k}^{i, b} \\
\bar{f}_{k}^{b} & =\bar{R} \bar{z}_{k}^{b}
\end{aligned}
$$

with

$$
\begin{aligned}
\bar{z}_{k}^{b} & =\left[\begin{array}{lll|llllll}
\beta_{k}^{i, 1} & \ldots & \beta_{k}^{i, n_{j}} & \xi_{k}^{i, 1} & \ldots & \xi_{k}^{i, b-1} & \xi_{k}^{i, b+1} & \ldots & \xi_{k}^{i, n_{j}}
\end{array}\right]^{T} \\
\bar{\delta}_{k}^{b} & =\left[\begin{array}{llllll}
\delta_{k}^{i, 1} & \ldots & \delta_{k}^{i, b-1} & \delta_{k}^{i, b+1} & \ldots & \delta_{k}^{i, n_{j}}
\end{array}\right]^{T}
\end{aligned}
$$

$\bar{A}=\left[\begin{array}{cc}\alpha I_{n_{j}} & 0 \\ 0 & I_{n_{j}-1}\end{array}\right], \bar{B}=(1-\alpha) I_{2 n_{j}-1}, \quad \bar{D}=\left[\begin{array}{c}0 \\ I_{n_{j}-1}\end{array}\right], \bar{C}=\left[\begin{array}{ll}I_{n_{j}} & I_{n_{j}-1}\end{array}\right], \bar{G}^{b}=\left[\begin{array}{c}0_{(b-1) \times 1} \\ 1 \\ 0_{\left(n_{j}-b\right) \times 1}\end{array}\right]$ and $\bar{R}=\left[\begin{array}{ll}0 & I_{n_{j}-1}\end{array}\right]$. Then, each model-based observer $b$ can be built as

$$
\begin{aligned}
\hat{\bar{z}}_{k+1}^{b} & =\bar{A} \hat{\bar{z}}_{k}^{b}+B \hat{u}_{k}+\bar{L}_{k}^{b}\left(y_{k}-\bar{C} \hat{\bar{z}}_{k}^{b}\right) \\
\hat{\bar{f}}_{k}^{b} & =\bar{R} \hat{\bar{z}}_{k}^{b}+\bar{K}_{k}^{b}\left(y_{k}-\bar{C} \hat{\bar{z}}_{k}^{b}\right)
\end{aligned}
$$

where $\bar{L}_{k}^{b}=\bar{L}^{b}\left(\theta_{k}\right)$ and $\bar{K}_{k}^{b}=\bar{K}^{b}\left(\theta_{k}\right)$ are the gain matrices of the observer (46) defined as

$$
\begin{array}{cc}
\bar{L}^{b}\left(\theta_{k}\right)=\bar{L}_{q}^{b} & \text { if } \theta_{k} \in \Theta^{(q)} \\
\bar{K}^{b}\left(\theta_{k}\right)=\bar{K}_{q}^{b} & \text { if } \quad \theta_{k} \in \Theta^{(q)}
\end{array}
$$

Define $\tilde{\bar{z}}^{b}=\bar{z}^{b}-\hat{\bar{z}}^{b}$ and $\tilde{\bar{f}}^{b}=\bar{f}^{b}-\hat{\bar{f}}^{b}$. It follows that

$$
\begin{aligned}
\tilde{\bar{z}}_{k+1}^{b} & =\left(\bar{A}-\bar{L}_{k}^{b} \bar{C}\right) \tilde{\bar{z}}_{k}^{b}+\bar{E} p_{k}+\bar{F} t_{k}+\bar{D} \bar{\delta}_{k}^{b}-\bar{L}_{k}^{b}\left(w_{k}+\bar{G}^{b} f_{k}^{i, b}\right) \\
\tilde{\bar{f}}_{k}^{b} & =\left(\bar{R}-\bar{L}_{k}^{b} \bar{C}\right) \tilde{\bar{z}}_{k}^{b}-\bar{K}_{k}^{b}\left(w_{k}+\bar{G}^{b} f_{k}^{i, b}\right)
\end{aligned}
$$

with $\bar{E}$ and $\bar{F}$ being built in analogy to $E$ and $F$. The exogenous signals affecting the observer $b$ are not only the fault generator $\bar{\delta}^{b}$, the noises $w$ and the unknown inputs $p$ and $t$ but also the fault $f^{i, b}$. We propose to omit the dependence of the error on the fault $f^{i, b}$ and to design the gain matrices of each observer (46) following the approach presented in Section 5.2 (the matrices $\bar{A}, \bar{C}, \bar{D}, \bar{E}, \bar{F}, \bar{L}^{b}$ and $\bar{K}^{b}$ in the place of the matrices $A, C, D$, $E, F, L$ and $K)$. Note that, in this case, the restriction regarding the CSE requirement of the $b$-th fault will not appear in the optimization problem (30). This extra design freedom is then used to achieve a greater minimization of the effect of the noises and the UIs on the fault estimates.

In the absence of the fault $f^{i, b}$, we can thus set the following decisions $\left(j=1, \ldots, n_{j}-1\right)$ for the $b$-th observer:

$$
\begin{cases}\text { if }\left|\hat{\bar{f}}_{k}^{b}[j]\right| \geq \bar{J}_{k}^{b}[j] & \text { Fault } \bar{f}^{b}[j] \\ \text { otherwise } & \text { No fault } \bar{f}^{b}[j]\end{cases}
$$

where $J^{b}[j]$ is designed following the strategies in Section 6.2. Note that the thresholds $\bar{J}^{b}[j]$ in (49) are smaller 


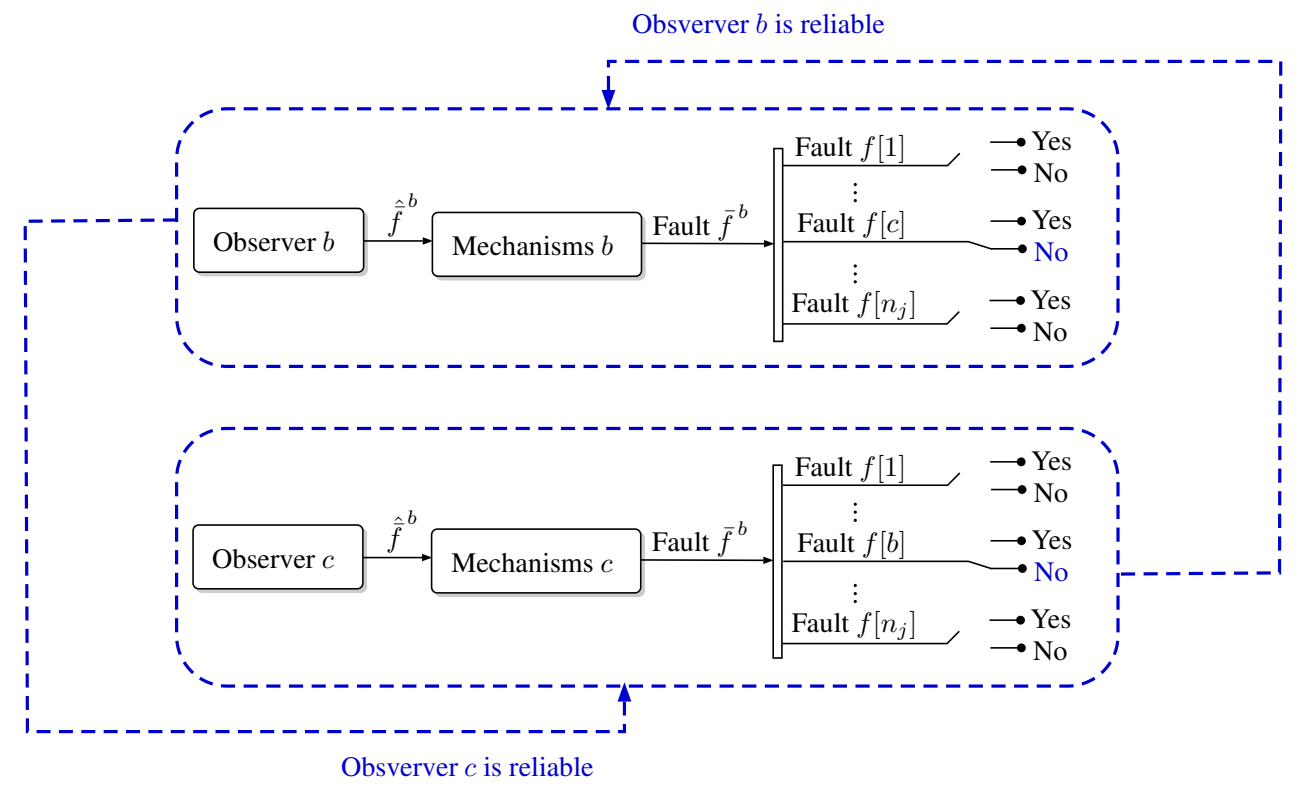

Figure 6. Reliability chain in the bank of observers and decision mechanisms.

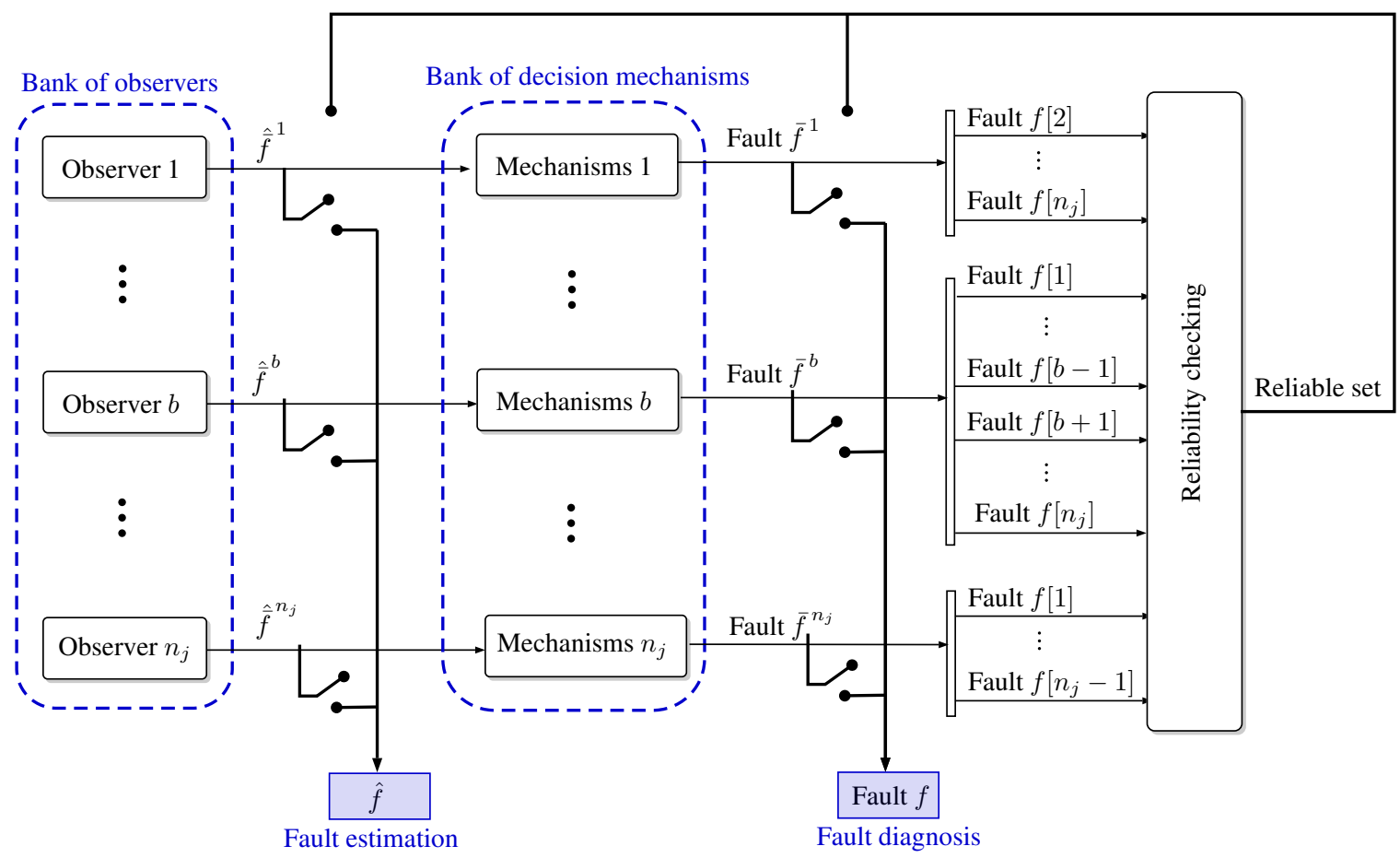

Figure 7. Bank of observers and decision mechanisms for FE and FD in each row of WTs $i$.

than the thresholds $J[j]$ in (36): when omitting the presence of the fault $f^{i, b}$, we achieve a greater attenuation from noises and UIs.

If the fault $f^{i, b}$ is present in the system, the decision mechanisms (49) which are based on the $b$-th observer are no longer reliable. Thus, an observer $b$ and the corresponding decision mechanisms are reliable if the absence of the fault $f^{i, b}$ is diagnosed by the decision mechanisms of at least one reliable observer $c(c \neq b)$. In turn, the reliability of $c$ implies that the decision mechanisms of the $b$-th observer diagnose the absence of the fault $f^{i, c}$, see Fig.6. In all, the proposed bank of observer and decision mechanisms enables FE and FD whenever 2 of the faults in a row $i$ are not present in the system (i.e., there are no more than $n_{j}-2$ simultaneous faults in a row). The estimation and diagnosis of the faults is made with any of the reliable observers and decision mechanisms of the bank. The strategy is summarized in Fig.7. 


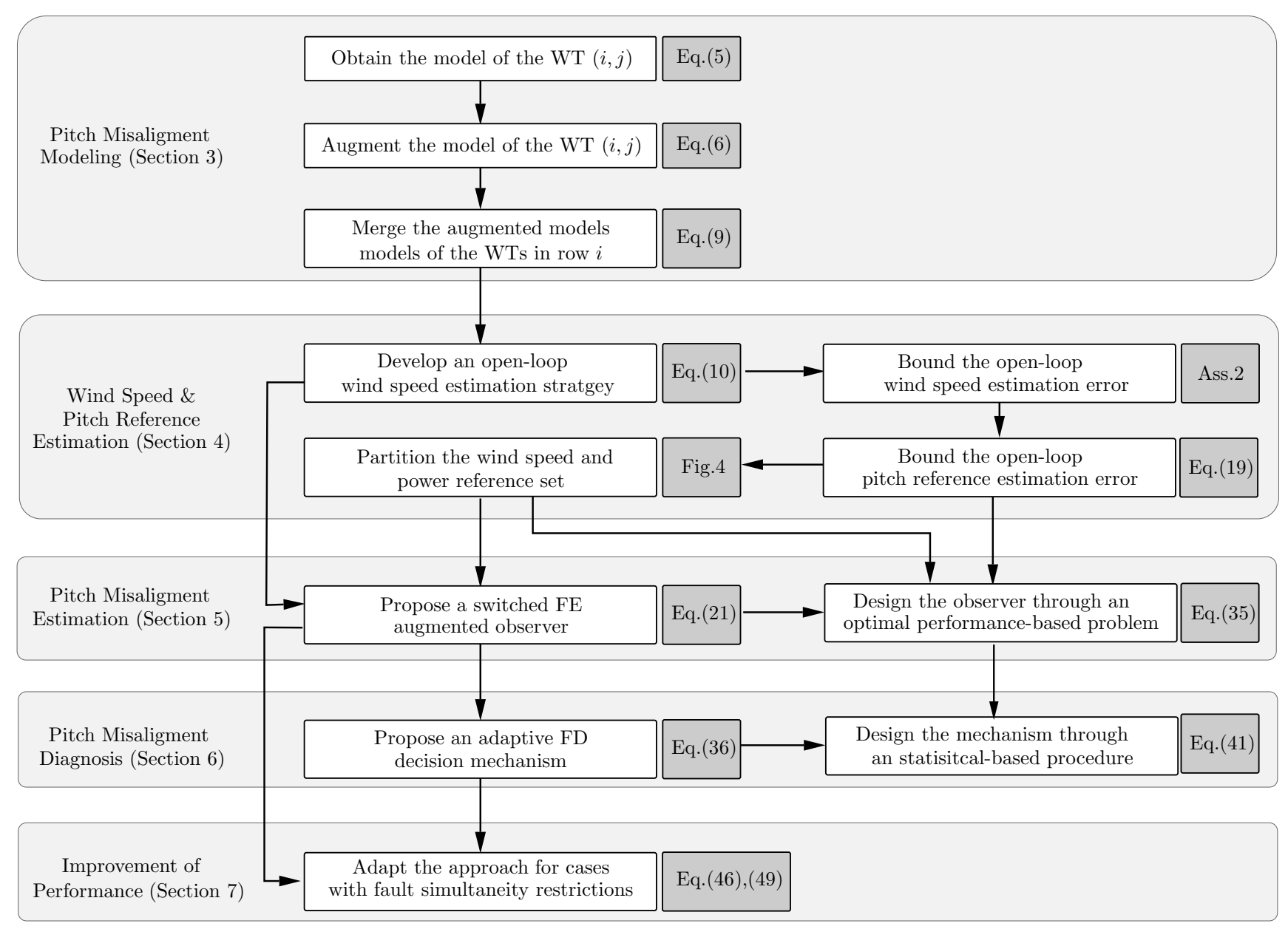

Figure 8. Summary of the proposed methodology.

\section{Benefits and Limitations of the Proposed Approach}

In the following, we compare the proposed methodology (summarized in Fig.8) to the solutions in relevant existing literature.

- The proposed approach utilizes a reduced number of measurements: the power reference, the collective pitch and the WS at the wind mast. This reduces the information needs w.r.t. other techniques such as the one in [22] (requiring also the generator speed measurement). It does not either require the information about the presumed fault size as it is required in [18].

- The residual-based techniques in [18-20] are focused on FD tasks. Contrariwise, the proposed approach is focused on both FE (Section 5) and FD (Section 6), being more suitable for AFTC strategies.

- The FD strategies in [18-20] are based on separate spatial and temporal residuals. In contrast, the proposed approach is based on a systematic multi-input multi-output (MIMO) observer that automatically merges the information acquired from both temporal and spatial inconsistencies. It thus optimally utilizes all the information acquired from the WF measurements.

- The proposed closed-loop approach is more actively robust against disturbances (i.e., uncertainties and noises) than the open-loop methods in [18-20].

- The switched system approach is a non-conservative procedure to handle the different levels of uncertainties along the WT operation range, leading to a less restrictive compromise between fault sensitivity and robustness.

- The proposed observer design in Section 5 guarantees certain local fault sensitivity with optimal disturbance rejection. This performance is not guaranteed in [18-20], where more ad-hoc and user knowledge-based tuning procedures are utilized to set this trade-off. 


\begin{tabular}{cccc}
\hline Fault ID & $0^{\circ}$ layout & $45^{\circ}$ layout & Time occurrence \\
\hline F1 & $(i=1, j=1)$ & $(i=1, j=3)$ & {$[1300,1400] \mathrm{s}$} \\
F2 & $(i=2, j=2)$ & $(i=3, j=3)$ & {$[3300,3400] \mathrm{s}$} \\
\hline
\end{tabular}

Table 2. Fault scenario description.

- The design does not consider the observer performance during the transitions between subsets. However, as indicated in Remark 7, if an appropriate gridding of the parameter set is obtained, these transitions are infrequent.

- The proposed observer design strategy entails certain computational cost. However, this design is performed offline and it does not affect the online computational burden of the FE and FD strategy (see Fig.5). Moreover, as indicated in Remark 7, if an appropriate gridding of the parameter set is obtained, this offline computational cost is considerably reduced.

- In contrast to the constant thresholds in $[18,22]$, the adaptive FD mechanisms presented in Section 6 allow the adjustment of the thresholds to the fault estimates along all the WT operation range. The proposed statisticalbased design of the FD thresholds provides tight bounds for the fault estimates obtained via switched observers and allows a rapid diagnosis of small faults.

- In this work, we also propose an extension of the proposed approach (Section 7) that allows an improvement of the FD performance for the cases in which not all the WTs become simultaneously faulty.

\section{Simulation Results}

The WF benchmark [17] includes a scenario of $4400 \mathrm{~s}$ in which different faults occur. In the first period between $0 \mathrm{~s}$ and $2300 \mathrm{~s}$, the WF cannot deliver the required power, while it can do so in the second period between $2300 \mathrm{~s}$ and $4400 \mathrm{~s}$. The collective pitch system of the WTs $(1,1)$ and $(2,2)$ of the $0^{\circ}$ layout are affected by misalignment of the blades causing an offset of $0.3^{\circ}$ in the pitch angle measurements, see Table 2 . In the following, we include the FE and FD results that we obtain with the proposed approach. All the observer designs are translated into multiobjective convex optimizations as detailed in Appendix B, set up in YALMIP [54] and solved using the solver MOSEK [55]. For simplification, we omit the obtained observer matrix gains $\left\{L_{q}, K_{q}\right\}_{\left\{1, \ldots, N_{\theta}\right\}}$ and the obtained bounds $\left\{\bar{p}_{q}, \bar{t}_{q}\right\}_{\left\{1, \ldots, N_{\theta}\right\}}$. Let us remark that in the figures of the section, unless explicitly stated otherwise, the fault estimates are in black and the thresholds of the diagnosis decisions are in red.

For the $0^{\circ}$ layout, we build a bank of observers and decision mechanisms for each row of WTs $(i=1, i=2$ and $i=3$ ) as explained in Section 7. Let us perform different observer designs with different values of the CSE requirements $\bar{\gamma}_{j}^{j}$ and let us use $\bar{\gamma}_{m}^{j}=\bar{\gamma}_{j}^{j} / 100$ for all $m \neq j$ in these designs (See Remark 6). If we denote as $\bar{\psi}^{j}$ the CSE requirement $\bar{\gamma}_{j}^{j}$ when taking into account the sampling time $T_{s}$ (i.e., $\bar{\psi}^{j}=\bar{\gamma}_{j}^{j} T_{s}$ ), we use $\bar{\psi}^{j}=[1,5,10,20,30,50]^{0^{2}} s$ for $j=1, \ldots, n_{j}$. The FAR criterion with $\bar{\phi}^{j}=1 \cdot 10^{-5}$ (i.e., one false alarm each 1000s) for $j=1, \ldots, n_{j}$ is used to compute the thresholds of the decision mechanisms. Fig.9 shows the effect of varying the CSE in the WT $(i=1, j=1)$, which is affected by the fault F1. As an example, the details on the results with $\bar{\psi}^{j}=30^{\circ^{2}} s$ are depicted in Fig.10. Fig.11 shows the results for the WT $(i=2, j=2)$, which is affected by the fault F2, and Fig.12 shows the results for the WT $(i=1, j=2)$, which operates in fault-free conditions. The results on the left hand side of the figures include the variables $\hat{f}[j]$ (black) and $J[j]$ (red) while the results on the right hand side compare the normalized variable $\hat{f}[j] / J[j]$ (black) with a unitary threshold (red).

Regardless of the value of the CSE requirement, all these figures (Fig.9, Fig.11 and Fig.12) show that when the power harvested from the wind is much lower than the power required by the WF controller, the achieved minimum diagnosable faults (MDFs) are smaller. In such cases, the estimated WS is low and the pitch reference estimation errors are small because for low WSs the pitch reference function is barely constant (see Fig. 2). Contrariwise, for high WSs the pitch reference function is monotonically increasing and the pitch estimation errors are larger. Let us now analyze the effect of the CSE requirement on the FE and FD performance. We verify that as the CSE 
Absolute variables $\left[{ }^{\circ}\right]$
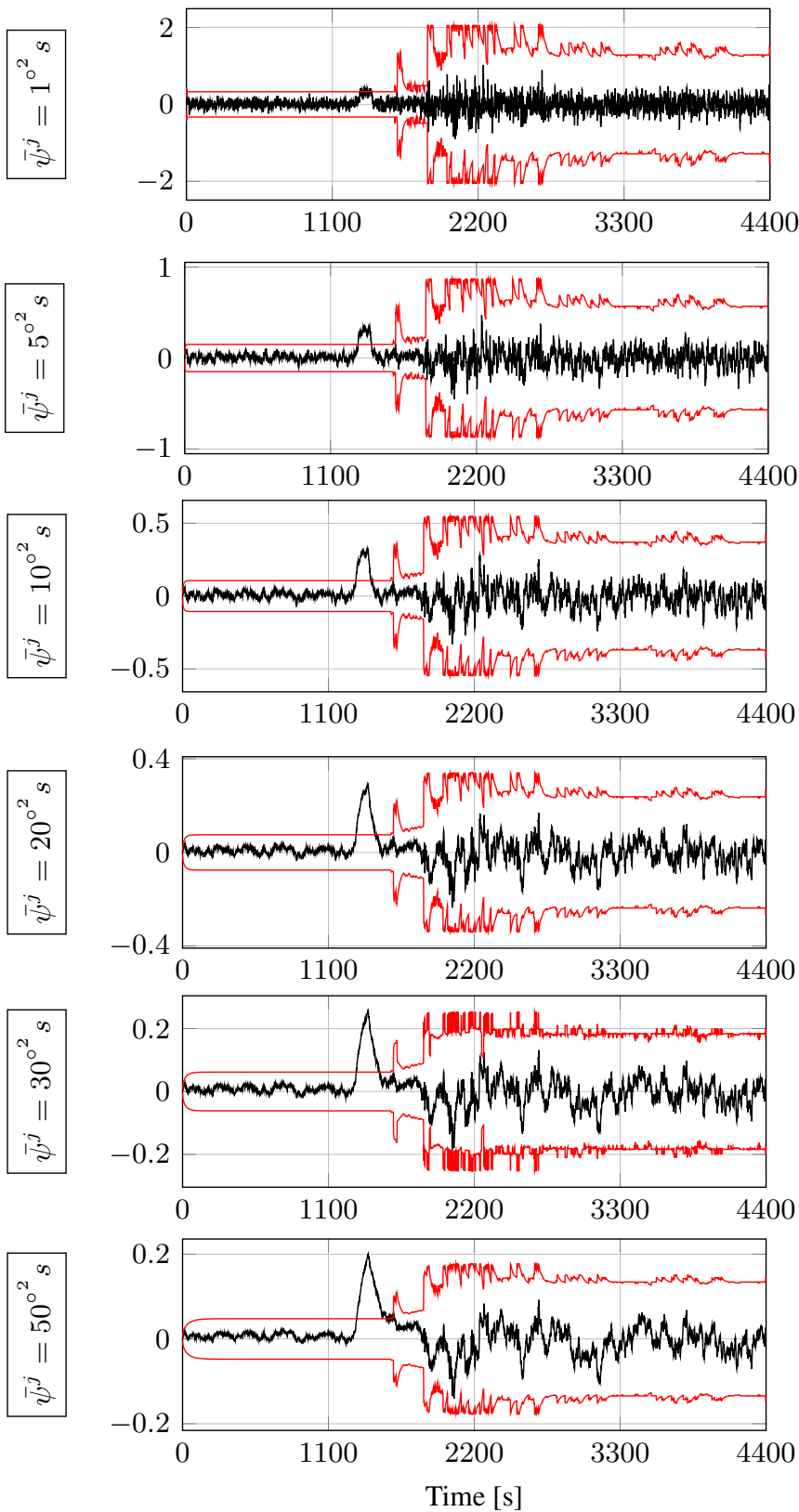

Normalized variables
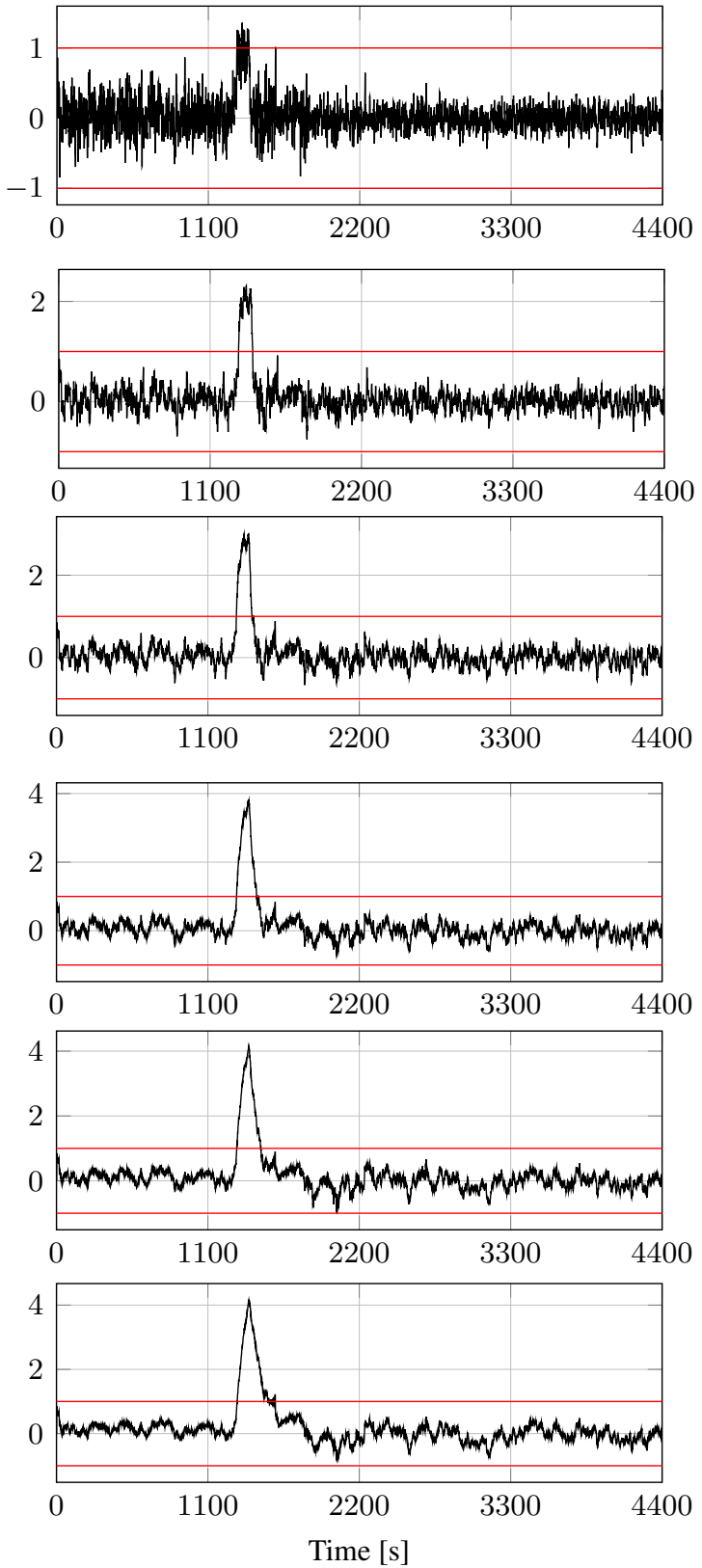

Figure 9. FE and FD with different CSE requirements $\bar{\psi}^{j}$. Turbine $(i=1, j=1)$ of the $0^{\circ}$ layout affected by the fault $\mathbf{F 1}$.

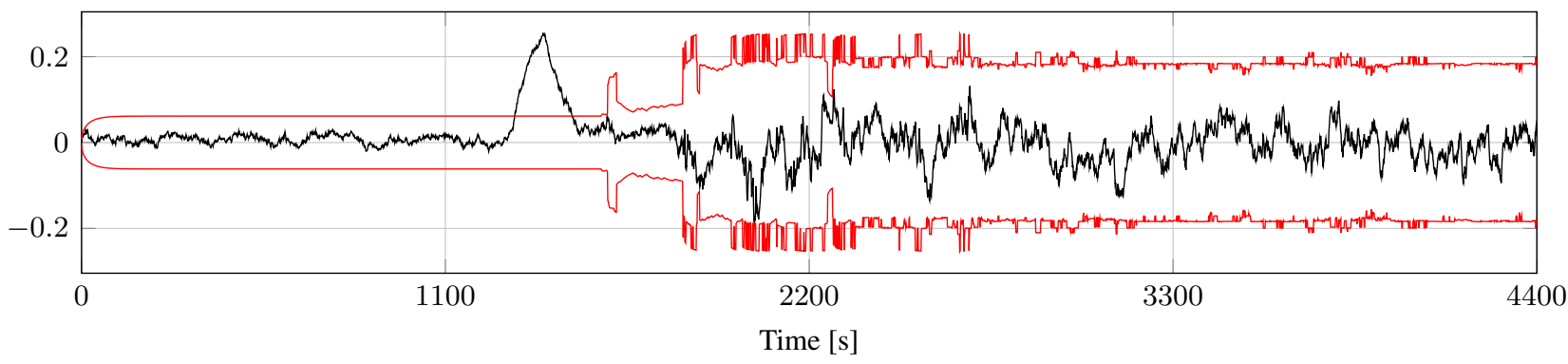

Figure 10. Details on the FE and FD results with a CSE requirement of $\bar{\psi}^{j}=30^{\circ}$ s. Turbine $(i=1, j=1)$ of the $0^{\circ}$ layout affected by the fault F1. Absolute variables in $\left[{ }^{\circ}\right]$.

requirement becomes more restrictive (i.e., $\bar{\psi}^{j}$ becomes smaller), the attenuation from the uncertainties and the noises diminishes and the minimum diagnosable faults (MDFs) are larger. On the other hand, the fault tracking 
Absolute variables $\left[{ }^{\circ}\right]$
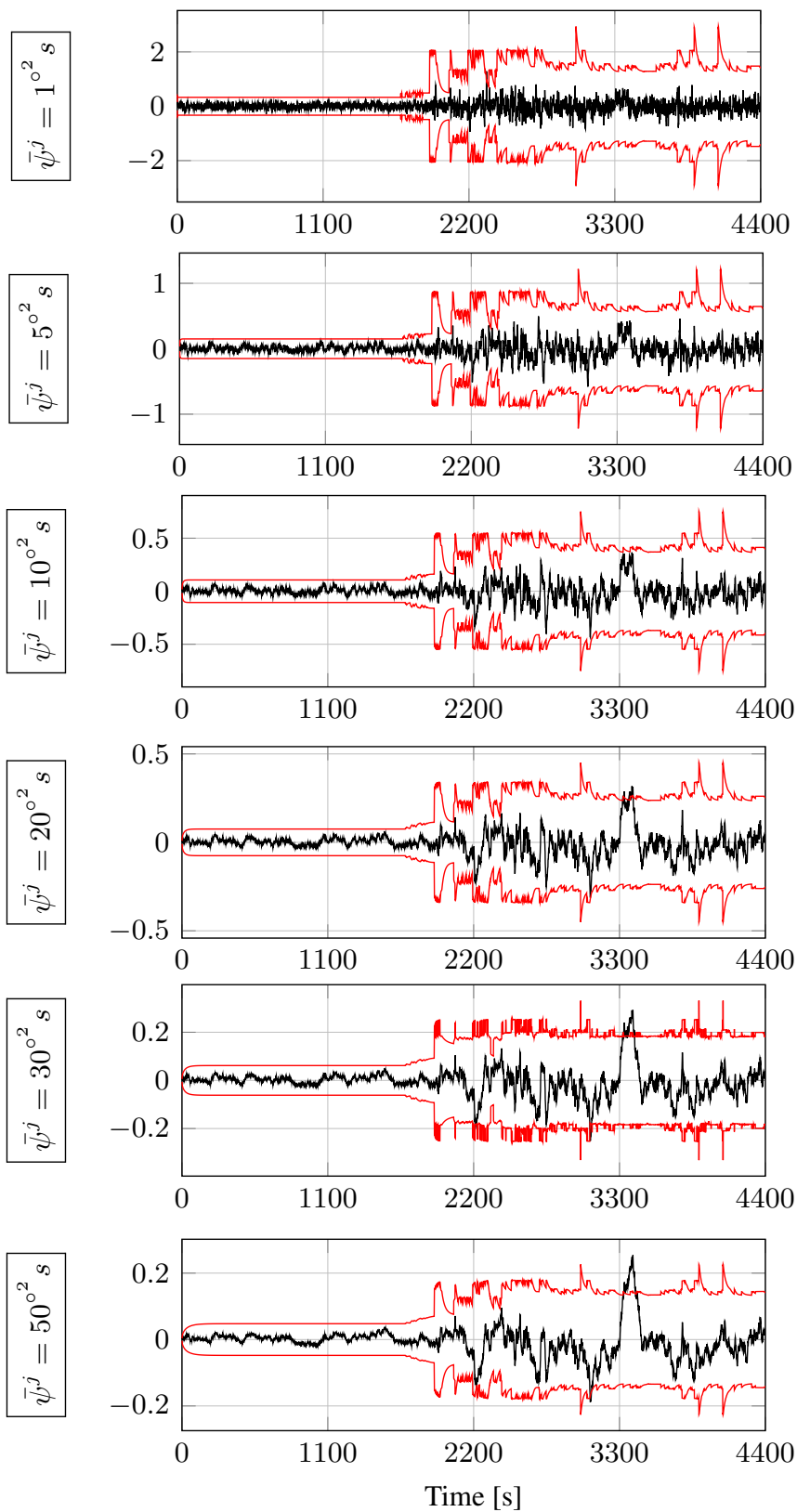

Normalized variables
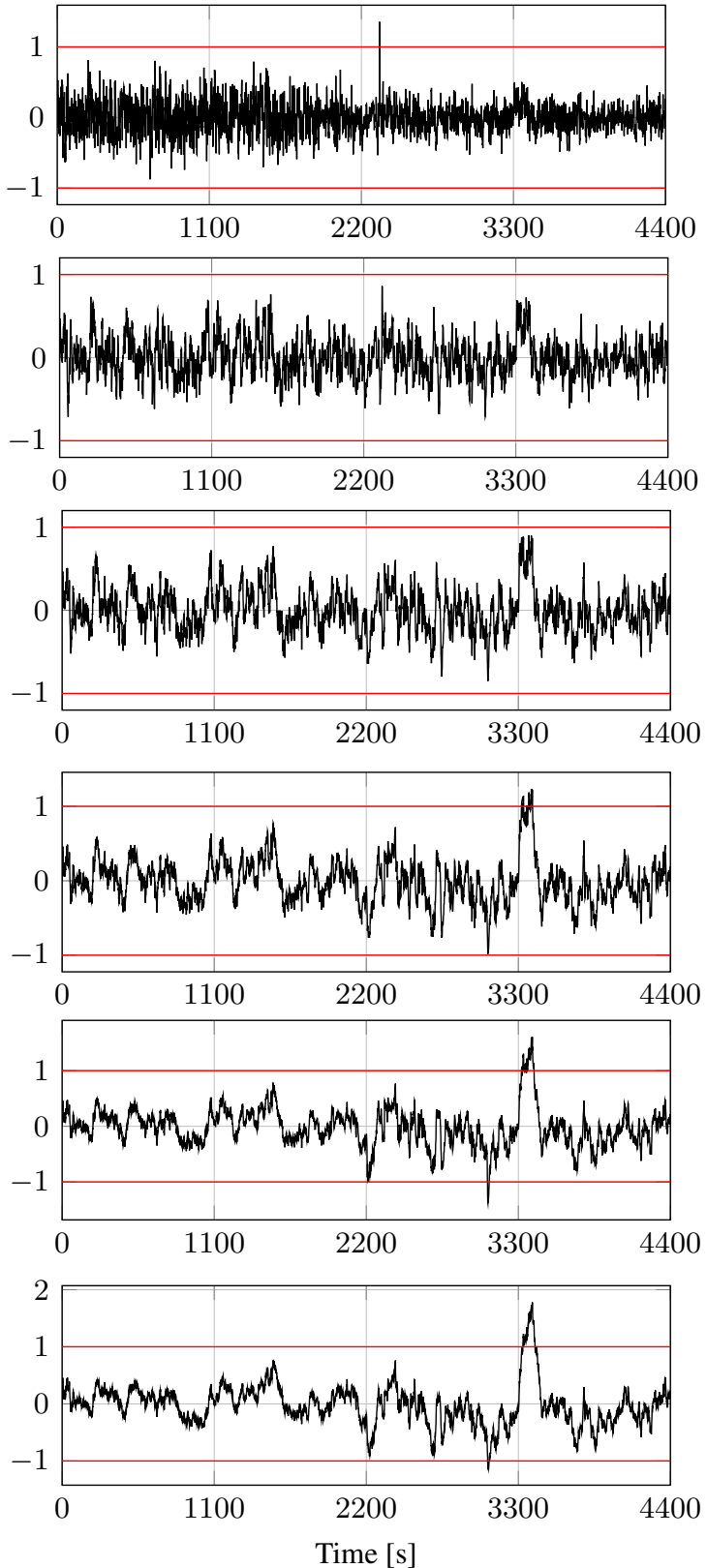

Figure 11. FE and FD with different CSE requirements $\bar{\psi}^{j}$. Turbine $(i=2, j=2)$ of the $0^{\circ}$ layout affected by the fault $\mathbf{F} 2$.

behaviour is improved and the detection times (DTs) diminish (as long as the achieved MDFs are smaller than the simulated faults).

Provided these deductions, it is reasonable that the fault F1, which occurs at the low WS period, is diagnosed with all the designed observers. In contrast, the fault F2, which occurs at the high WS period, is only diagnosed when using the designs with the less restrictive CSE requirements. Fig.13 compares the diagnosis of the fault $\mathbf{F 1}$ and of the fault $\mathbf{F 2}$ provided by three designs with a restrictive, an intermediate and a moderate CSE requirement (i.e., $\bar{\psi}^{j}=5^{\circ^{2}} s, \bar{\psi}^{j}=20^{\circ^{2}} s$ and $\bar{\psi}^{j}=50^{\circ 2} s$ ). For ease of comparison, the figure includes the normalized variables $\hat{f}[j] / J[j]$ of these three cases. Thus, bigger differences between the value of the variable $\hat{f}[j] / J[j]$ and the unitary threshold imply smaller MDFs. The DT of the fault F1 is smaller if we use an observer designed with a smaller CSE requirement; in contrast, the difference between the normalized variable $\hat{f}[j] / J[j]$ and the unitary threshold becomes smaller. For the fault the fault F2, we prove that FD is not achieved if the restrictive CSE requirement is used in the design. The DTs of the other two designs are similar: even if $\bar{\psi}^{j}=20^{\circ}{ }^{2} s$ is smaller than $\bar{\psi}^{j}=50^{\circ} s$, 
Absolute variables $\left[{ }^{\circ}\right]$
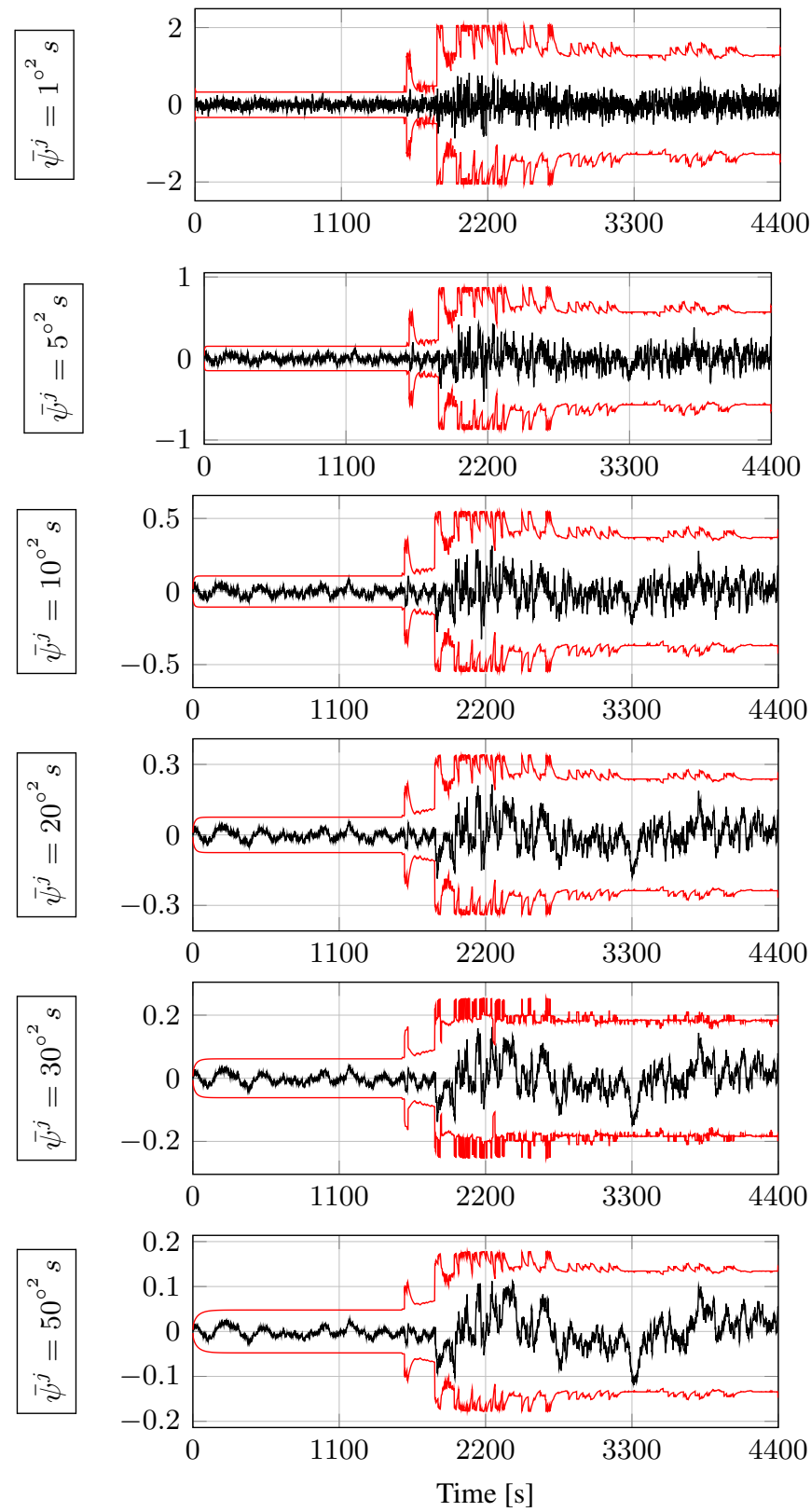

Normalized variables
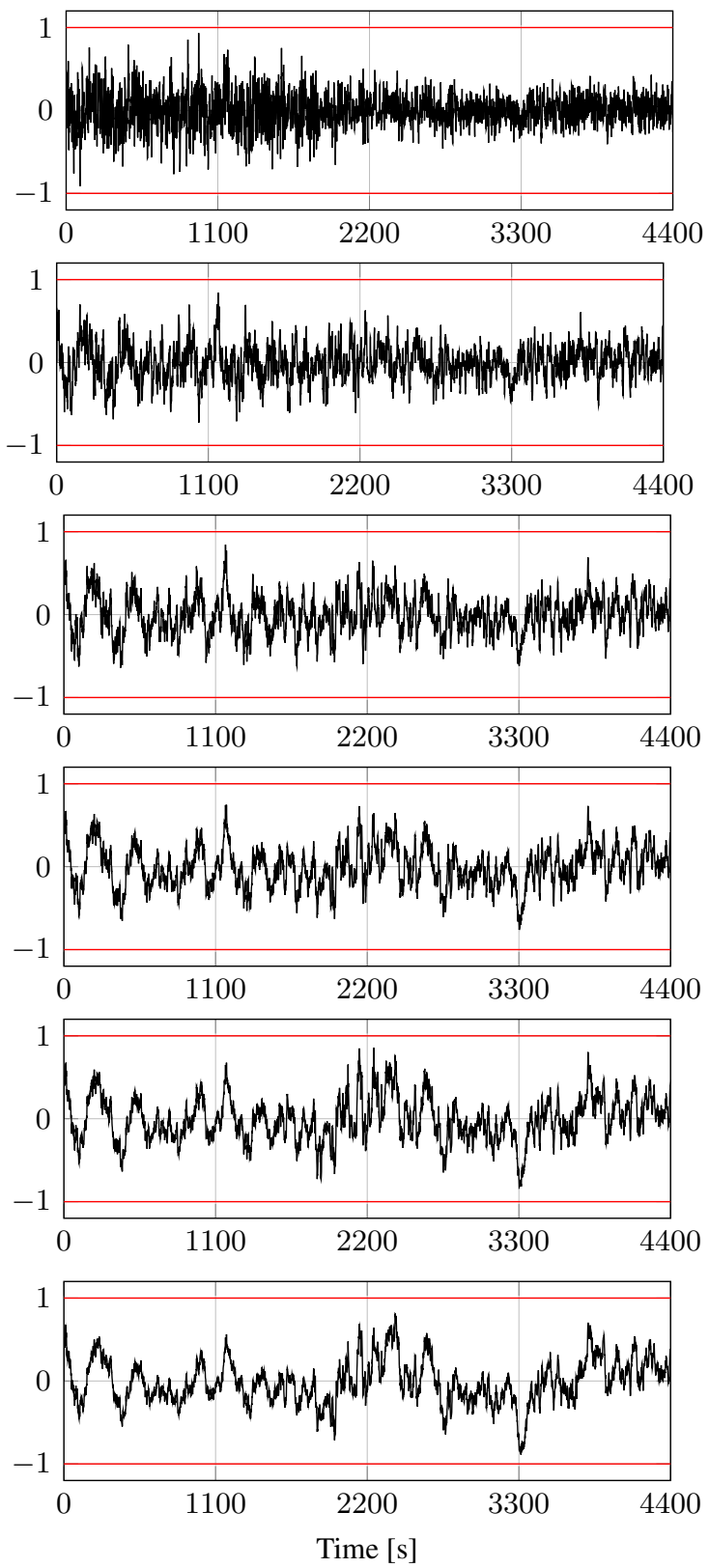

Figure 12. FE and FD with different CSE requirements $\bar{\psi}^{j}$. Turbine $(i=1, j=2)$ of the $0^{\circ}$ layout in fault-free conditions.

the achieved MDF at $3300 \mathrm{~s}$ for $\bar{\psi}^{j}=20^{\circ} s$ is very close to $0.30^{\circ}$ (see Table 3 ) and FD is compromised. In contrast, the achieved MDF for $\bar{\psi}^{j}=50^{\circ} s$ at $3300 \mathrm{~s}$ is $0.14^{\circ}$. Table 3 summarizes these results for all the designs. Let us remark that the maximum values of the MDFs are bigger at the row $i=2$ than at the row $i=1$ because the pitch reference estimation errors are higher as the distance to the wind mast increases. At $1300 \mathrm{~s}$ and $3300 \mathrm{~s}$ these differences are coincidentally minimal. The biggest differences appear at the WSs and power references for which the slope of the pitch reference function is more pronounced. Finally, note that the diagnosis of the fault F1 is achieved with the design of $\bar{\psi}^{j}=1^{{ }^{2}} s$ even if the MDF at $1300 \mathrm{~s}$ is bigger than the fault size (i.e., $0.33^{\circ}$ vs. $0.30^{\circ}$ ). The effect of the noises and uncertainties enhances this diagnosis; however, the fault estimate oscillates around the threshold of the diagnosis decision and the results are thus misleading in this case.

In the following, we use $\bar{\psi}^{j}=30^{\circ} \mathrm{s}$, and $\bar{\phi}^{j}=1 \cdot 10^{-5}\left(j=1, \ldots, n_{j}\right)$ in the designs. Fig.14 and Fig. 15 show the results for all the WTs in the WF when we analyze the $0^{\circ}$ layout. To enhance the comprehension of the functioning of the bank of observers and decision mechanisms, let us study each row of WTs in the WF. In the row $i=1$, 
$(i=1, j=1)$

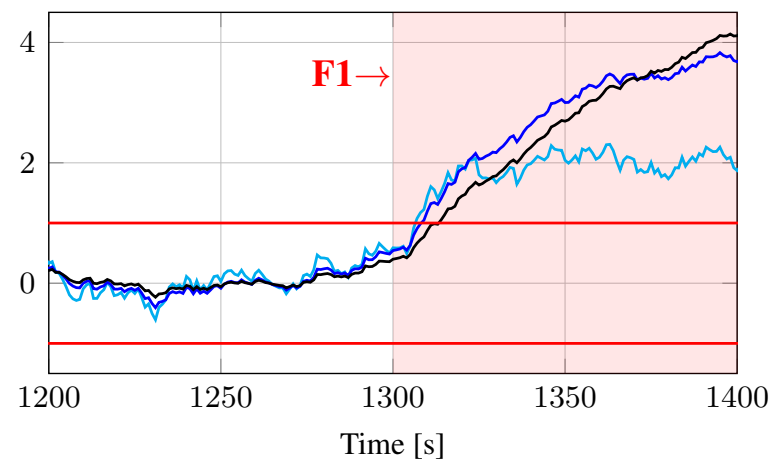

$(i=2, j=2)$

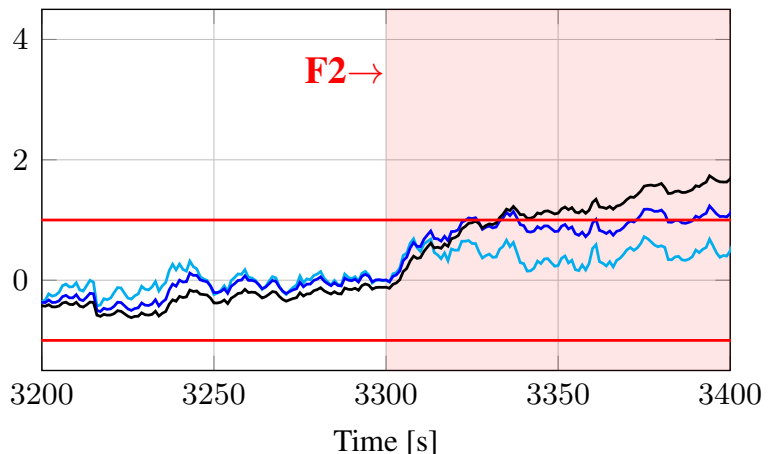

$-\bar{\psi}^{j}=5^{\circ^{2}} s-\bar{\psi}^{j}=20^{\circ^{2}} s-\bar{\psi}^{j}=50^{\circ} s$

Figure 13. Comparison of FE and FD performance with different CSE requirements ( $0^{\circ}$ layout). Normalized variables.

\begin{tabular}{|c|c|c|c|c|c|c|c|c|}
\hline \multirow{2}{*}{$\operatorname{CSE}\left[0^{2} s\right]$} & \multicolumn{2}{|c|}{$\mathrm{MDF}$ at $1300 \mathrm{~s}\left[^{\circ}\right]$} & \multicolumn{2}{|c|}{$\mathrm{MDF}$ at $3300 \mathrm{~s}\left[{ }^{\circ}\right]$} & \multicolumn{2}{|c|}{ largest MDF [ $\left.{ }^{\circ}\right]$} & \multicolumn{2}{|c|}{ DT $[s]$} \\
\hline & $i=1$ & $i=2$ & $i=1$ & $i=2$ & $i=1$ & $i=2$ & F1 & F2 \\
\hline 1 & 0.33 & 0.33 & 1.28 & 1.31 & 2.07 & 2.93 & 10 & - \\
\hline 5 & 0.15 & 0.15 & 0.57 & 0.58 & 0.88 & 1.22 & 7 & - \\
\hline 10 & 0.11 & 0.11 & 0.37 & 0.38 & 0.55 & 0.75 & 8 & - \\
\hline 20 & 0.08 & 0.08 & 0.24 & 0.24 & 0.34 & 0.45 & 9 & 23 \\
\hline 30 & 0.06 & 0.06 & 0.18 & 0.18 & 0.25 & 0.33 & 10 & 24 \\
\hline 50 & 0.05 & 0.05 & 0.13 & 0.14 & 0.18 & 0.23 & 14 & 33 \\
\hline
\end{tabular}

Table 3. Comparison of diagnosis performance with different observers $\left(0^{\circ}\right.$ layout $)$.

between $1300 \mathrm{~s}$ and $1400 \mathrm{~s}$, the observer $b=1$ and the corresponding decision mechanisms are corrupted by the appearance of the fault F1 in the WT $(i=1, j=1)$ (i.e., $f_{k}^{1,1} \neq 0$ if $\left.k \in[1300,1400]\right)$. The estimates of the fault $f^{1,1}$ provided by the observers $b=2$ and $b=3$ indicate it. Thus, FE and FD is achieved with the results of $b=2$ and $b=3$. Similarly, in the row $i=2$, between $3300 \mathrm{~s}$ and $3400 \mathrm{~s}$, the results are provided by either $b=1$ or $b=3$ because $b=2$ is corrupted by the fault $\mathbf{F 2}$ in the WT $(i=2, j=2)$. Fig.16 includes the results of the non-reliable observer $b=2$ and the corresponding decision mechanism. They estimate and diagnose a non-existent fault in the WT $(i=2, j=3)$. Fortunately, the estimates provided by the observers $b=1$ and $b=3$ are reliable and FD is achievable in the WF (Fig.14 and Fig.15).

Let us now design a single observer and a single set of decision mechanisms for each row of WTs of the $0^{\circ}$ layout. Fig.17 includes the results for the WTs in the column $j=1$ when the bank of observers and decision mechanisms is not used. The MDFs at the low WS period do not vary significantly. Contrariwise, at the high WS period, the MDFs increase drastically and this difference becomes bigger as the distance to the wind mast increases. Thus, we deduce that estimation and diagnosis are more difficult when it is not possible to build a bank of observers and decision mechanisms. This is the case where the wind direction is such that there are less than three WTs in a row (e.g., the WTs in the rows $i=1,2,4,5$ of the $45^{\circ}$ layout for which we do not include the results due to space constraints). In such cases, the FE and FD results are similar to the results in Fig.17 . A possible solution to mitigate this effect is to provide the WTs with laser anemometers (LIDAR), which offer a method of remote WS measurement and thus, the wind estimation errors decrease [56].

Finally, Fig.18 shows the estimation results when other densities are utilized for gridding the parameter set $\Theta$. We prove that if the number of intervals $N_{\nu}$ and $N_{p}$ is reduced, the offline computational burden decreases at the cost of some performance conservatism in terms of MDFs. In these cases, fewer changes of subset membership occur and the assumption of infrequent membership switches, which is used to design the FD thresholds, is more realistic. In any case, the chosen number of intervals (i.e., $N_{\nu}=N_{p}=7$ ) has proved to satisfy an adequate 

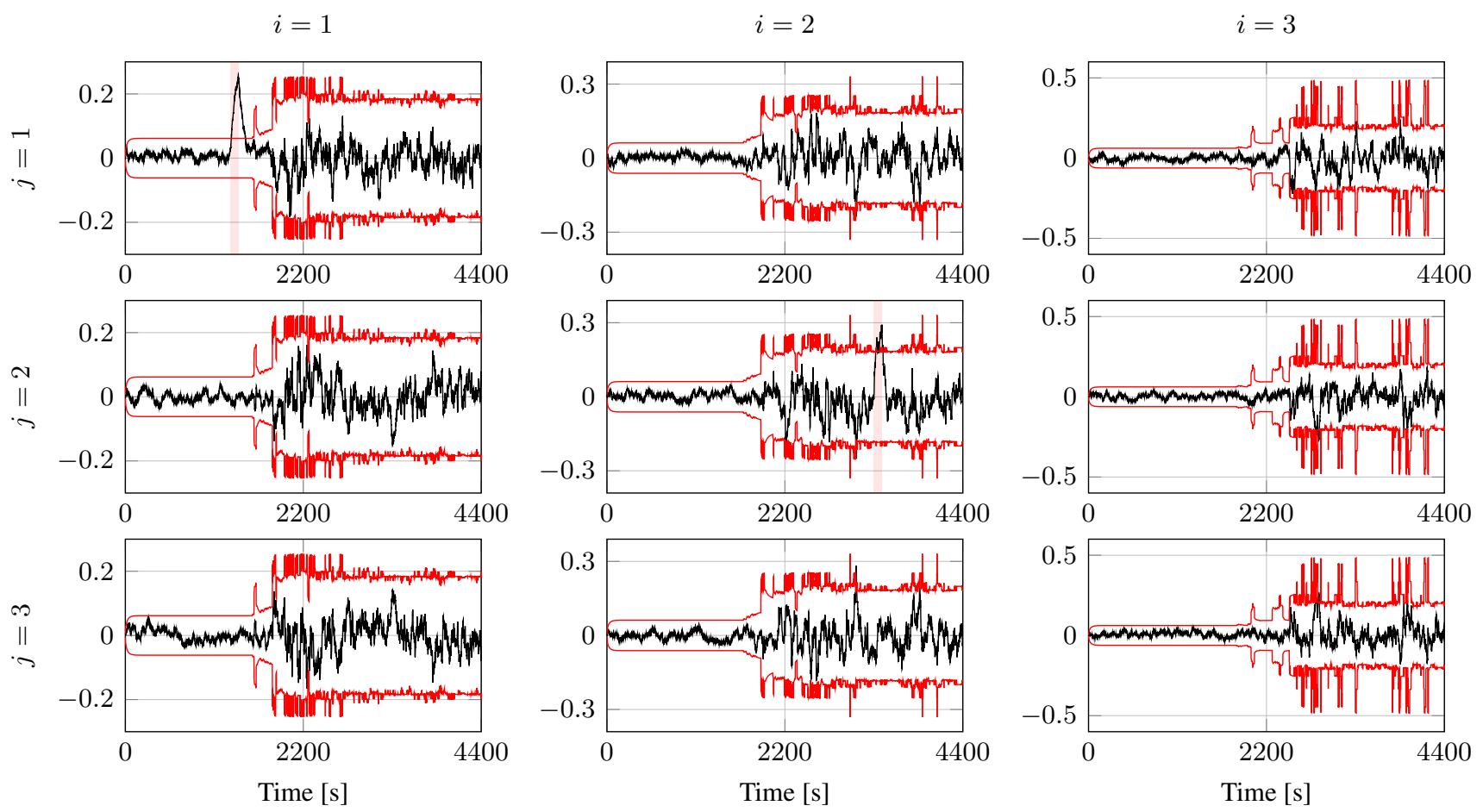

Figure 14. FE and FD with $\bar{\psi}^{j}=30^{\circ} \mathrm{s}\left(0^{\circ}\right.$ layout $)$. Absolute variables in $\left[{ }^{\circ}\right]$. $i=1$
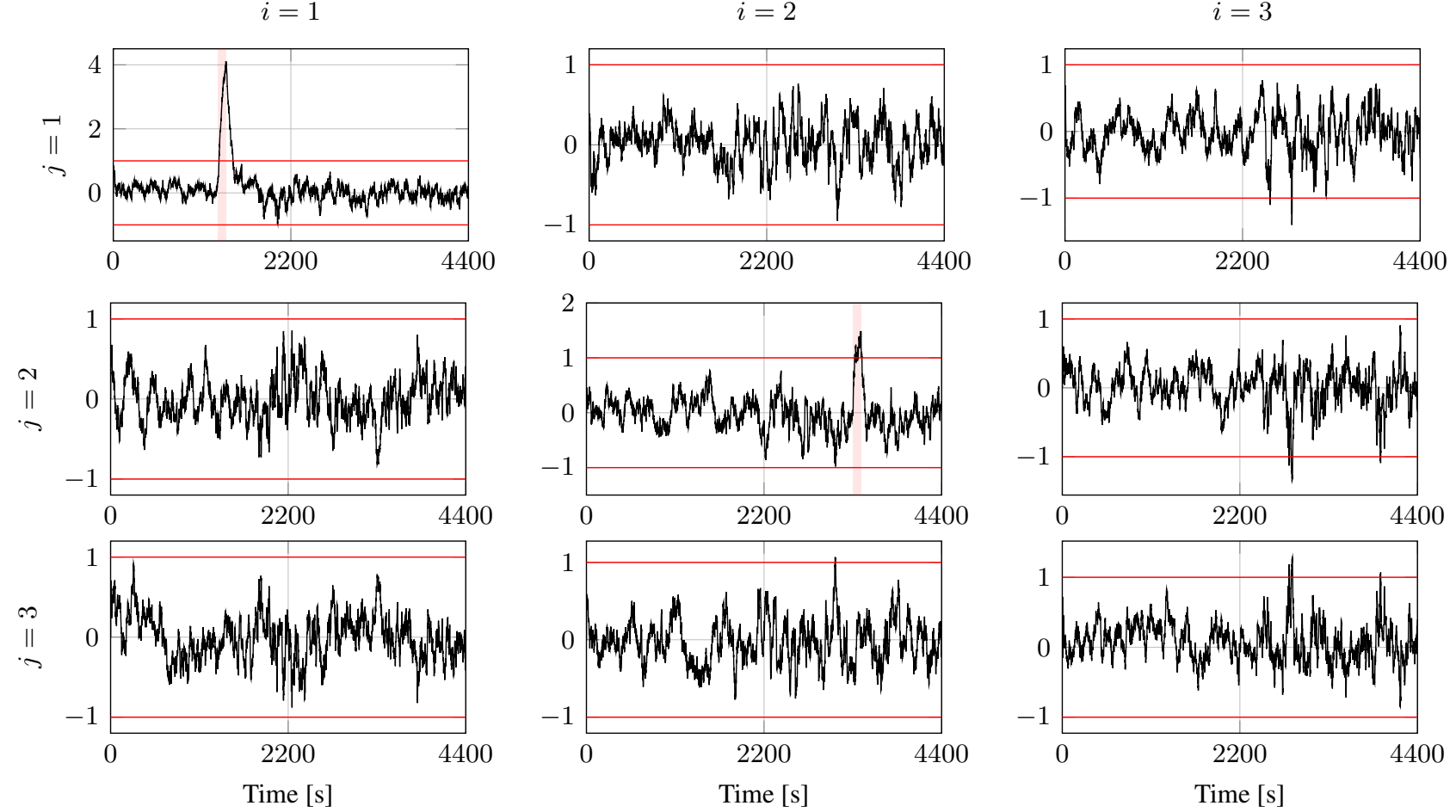

Figure 15. FE and FD with $\bar{\psi}^{j}=30^{\circ}{ }^{2} \mathrm{~s}\left(0^{\circ}\right.$ layout $)$. Normalized variables.

trade-off between computational cost and performance conservatism. Moreover, as show in the figures included in this section, few significant bound changes occur along the simulation of 4400s. Hence, we deduce that there are infrequent membership switches between intervals with significantly different error bounds. 
$b=1$

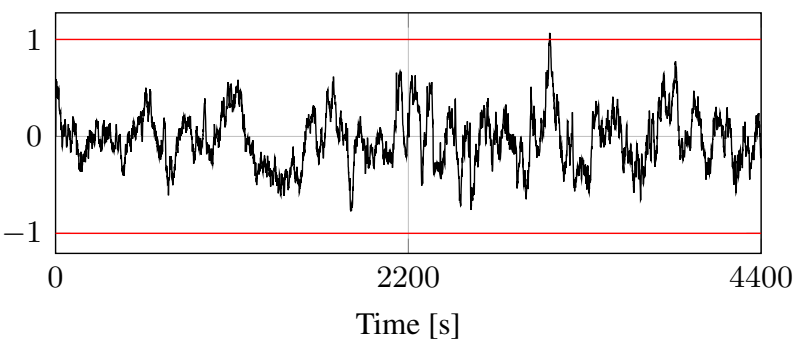

$b=2$

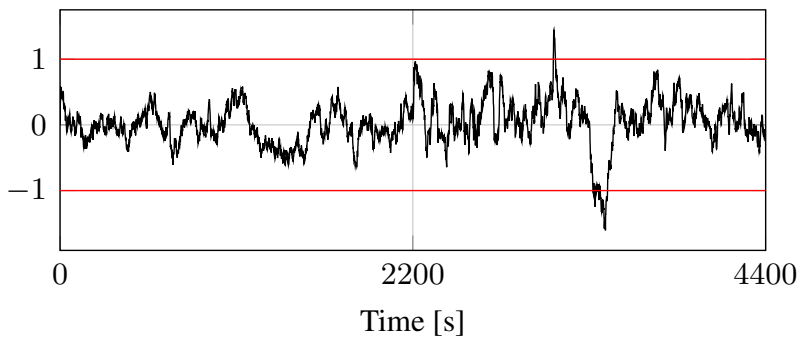

Figure 16. Reliability of the observers and decision mechanisms, $\bar{\psi}^{j}=30^{\circ}\left(0^{\circ}\right.$ layout, $\left.i=2, j=3\right)$. Normalized variables.

$i=1$

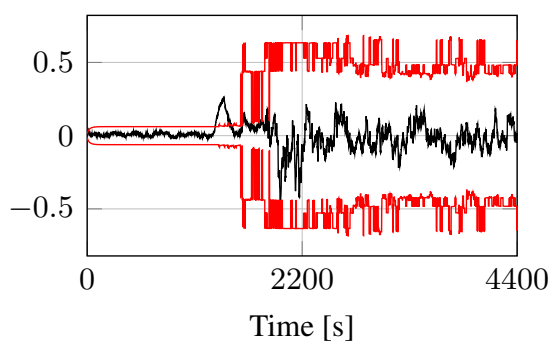

$i=2$

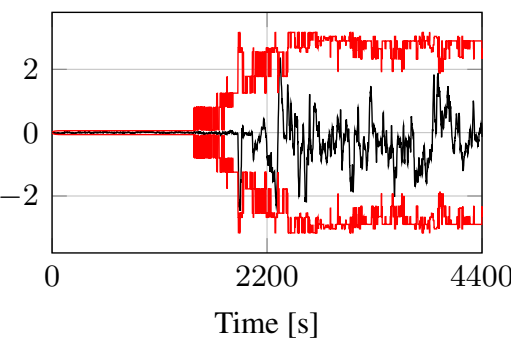

$i=3$

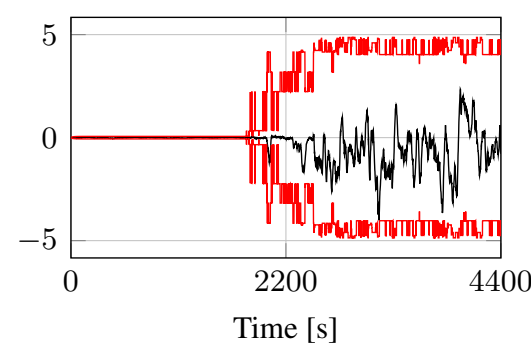

Figure 17. FE and FD without fault simultaneity restrictions, $\bar{\psi}^{j}=30^{\circ}\left(0^{\circ}\right.$ layout, $\left.j=1\right)$. Absolute variables in [ $\left.{ }^{\circ}\right]$.

$N_{p}=N_{\nu}=1$

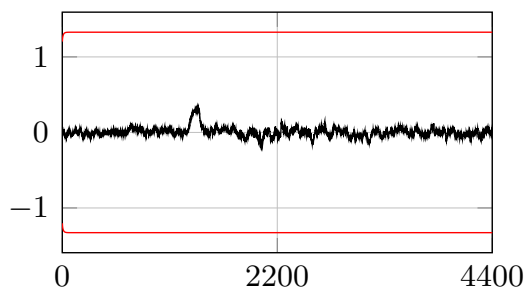

$N_{p}=N_{\nu}=2$

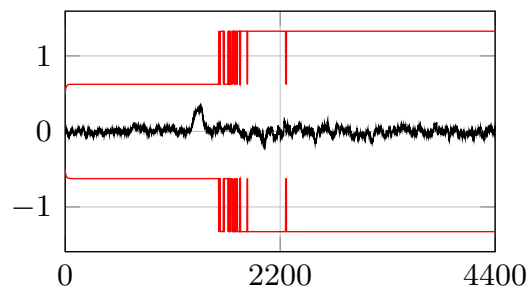

$N_{p}=N_{\nu}=7$

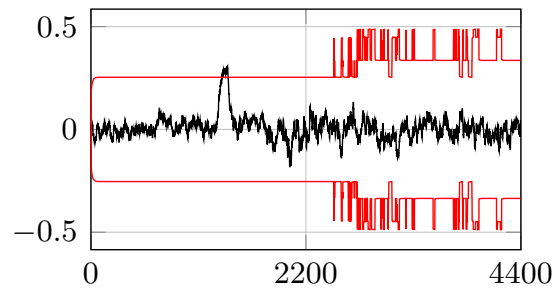

Figure 18. FE and FD with different gridding densities, $\bar{\psi}^{j}=30^{\circ} \mathrm{s}\left(0^{\circ}\right.$ layout, $\left.i=1, j=1\right)$. Absolute variables in $\left[{ }^{\circ}\right]$.

\section{Conclusion}

In this paper a model-based closed-loop FE and FD strategy is proposed for the pitch misalignments of the WTs in a WF. The FE algorithm includes a switched observer that is designed offline to guarantee global stability and local steady-state performance. The FE performance is intuitively fixed in the proposed design. The fault estimates are then used in statistical-based decision mechanisms for FD. The adaptive thresholds of these mechanisms are computed online under the FAR criterion. Under certain restrictions regarding the number of simultaneous faulty WTs, we improve the performance of the FE and FD strategy using a bank of observers and decision mechanisms.

The simulations performed on the WF benchmark demonstrate that the proposed strategy is suitable to estimate and diagnose pitch misalignments. The proposed spatially and temporally integrated strategy allows estimating and diagnosing pitch misalignments in the order of $0.1^{\circ}$ at low wind speeds and in the order of $1^{\circ}$ at high wind speeds. It should be emphasized that the proposed strategy can be extended for FE and FD in other WT subsystems at a WF level. Future research will include this extension. For its part, the use of the pitch misalignment estimates in individual fault tolerant control highlights as immediate future work. 


\section{Acknowledgements}

This work has been supported by the Spanish Ministry of Education, Culture and Sports (Grant FPU14/01592),

Jaume I of Castelló (Project P11B2015-42).

\section{References}

[1] Omar Ellabban, Haitham Abu-Rub, and Frede Blaabjerg. Renewable energy resources: Current status, future prospects and their enabling technology. Renewable and Sustainable Energy Reviews, 39:748-764, 2014.

[16] Josh Kusnick, Douglas E Adams, and Daniel T Griffith. Wind turbine rotor imbalance detection using nacelle and blade measurements. Wind Energy, 18(2):267-276, 2015.

[17] Peter F Odgaard and Jakob Stoustrup. Fault tolerant wind farm control - A benchmark model. In Proceedings of the IEEE International Conference on Control Applications (CCA), 2013, pages 412-417. IEEE, 2013. 
[18] Anders B Borcehrsen, Jesper A Larsen, and Jakob Stoustrup. Fault detection and load distribution for the wind farm challenge. IFAC Proceedings Volumes, 47(3):4316-4321, 2014.

[19] Joaquim Blesa, Pedro Jiménez, Damiano Rotondo, Fatiha Nejjari, and Vicenç Puig. An interval NLPV parity equations approach for fault detection and isolation of a wind farm. IEEE Transactions on Industrial Electronics, 62(6):37943805, 2015.

[20] Eric Duviella, Lisa Serir, and Moamar Sayed-Mouchaweh. An evolving classification approach for fault diagnosis and prognosis of a wind farm. In Proceedings of the 2nd Conference on Control and Fault-Tolerant Systems (SysTol), 2013, pages 377-382. IEEE, 2013.

[21] Silvio Simani, Saverio Farsoni, and Paolo Castaldi. Residual generator fuzzy identification for wind turbine benchmark fault diagnosis. Machines, 2(4):275-298, 2014.

[22] Silvio Simani, Paolo Castaldi, and Saverio Farsoni. Data-driven fault diagnosis of a wind farm benchmark model. Energies, 10(7):866, 2017.

[23] Youmin Zhang and Jin Jiang. Bibliographical review on reconfigurable fault-tolerant control systems. Annual Reviews in Control, 32(2):229-252, 2008.

[24] Zafer Civelek, Murat Lüy, Ertuğrul Çam, and Hayati Mamur. A new fuzzy logic proportional controller approach applied to individual pitch angle for wind turbine load mitigation. Renewable Energy, 111:708-717, 2017.

[25] Ke Zhang, Bin Jiang, Peng Shi, and Vincent Cocquempot. Observer-based fault estimation techniques. Springer Science \& Business Media, 2018.

[26] Zhiwei Gao, Carlo Cecati, and Steven X Ding. A survey of fault diagnosis and fault-tolerant techniques - Part I: Fault diagnosis with model-based and signal-based approaches. IEEE Transactions on Industrial Electronics, 62(6):3757$3767,2015$.

[27] Zhendong Sun. Switched linear systems: Control and design. Springer Science \& Business Media, 2006.

[28] Ester Sales-Setién and Ignacio Peñarrocha-Alós. Multiobjective performance-based designs in fault estimation and isolation for discrete-time systems and its application to wind turbines. International Journal of Systems Science, pages $1-23,2019$.

[29] Asier González-González, Alberto Jimenez Cortadi, Diego Galar, and Lorenzo Ciani. Condition monitoring of wind turbine pitch controller: A maintenance approach. Measurement, 123:80-93, 2018.

[30] Hamed Badihi, Youmin Zhang, and Henry Hong. Active power control design for supporting grid frequency regulation in wind farms. Annual Reviews in Control, 40:70-81, 2015.

[31] Torben Knudsen, Thomas Bak, and Mikael Svenstrup. Survey of wind farm control-power and fatigue optimization. Wind Energy, 18(8):1333-1351, 2015.

[32] Anca D Hansen, Poul Sørensen, Florin Iov, and Frede Blaabjerg. Centralised power control of wind farm with doubly fed induction generators. Renewable Energy, 31(7):935-951, 2006.

[33] Ke Zhang, Bin Jiang, Vincent Cocquempot, and Huaguang Zhang. A framework of robust fault estimation observer design for continuous-time/discrete-time systems. Optimal Control Applications and Methods, 34(4):442-457, 2013.

[34] Ester Sales-Setien and Ignacio Peñarrocha. Trade-offs on fault estimation via proportional multiple-integral and multiple-resonant observers for discrete-time systems. IET Control Theory \& Applications, 13(5):659-671, 2019.

[35] Zhiwei Gao. Fault estimation and fault-tolerant control for discrete-time dynamic systems. IEEE Transactions on Industrial Electronics, 62(6):3874-3884, 2015.

[36] Ai-Guo Wu, Gang Feng, and Guang-Ren Duan. Proportional multiple-integral observer design for discrete-time descriptor linear systems. International Journal of Systems Science, 43(8):1492-1503, 2012.

[37] Torben Knudsen, Thomas Bak, and Mohsen Soltani. Prediction models for wind speed at turbine locations in a wind farm. Wind Energy, 14(7):877-894, 2011.

[38] Zedjiga Yacine, Dalil Ichalal, Naïma Ait-Oufroukh, Saïd Mammar, and Saïd Djennoune. Takagi-Sugeno observers: experimental application for vehicle lateral dynamics estimation. IEEE Transactions on Control Systems Technology, 23(2):754-761, 2015. 
[39] Suneel K Kommuri, Michael Defoort, Hamid R Karimi, and Kalyana C Veluvolu. A robust observer-based sensor fault-tolerant control for PMSM in electric vehicles. IEEE Transactions on Industrial Electronics, 63(12):7671-7681, 2016.

[40] Zhiwei Gao, Xiaoxu Liu, and Michael ZQ Chen. Unknown input observer-based robust fault estimation for systems corrupted by partially decoupled disturbances. IEEE Transactions on Industrial Electronics, 63(4):2537-2547, 2016.

[41] Ester Sales-Setién, Ignacio Peñarrocha, Daniel Dolz, and Roberto Sanchis. Performance-based design of PI observers for fault diagnosis in LTI systems under gaussian noises. In Proceedings of the 3rd Conference on Control and FaultTolerant Systems (SysTol), 2016, pages 407-412. IEEE, 2016.

[42] Ping Zhang and Steven X Ding. An integrated trade-off design of observer based fault detection systems. Automatica, 44(7):1886-1894, 2008.

[43] Jamal Daafouz, Pierre Riedinger, and Claude Iung. Stability analysis and control synthesis for switched systems: a switched Lyapunov function approach. IEEE Transactions on Automatic Control, 47(11):1883-1887, 2002.

[44] Hai Lin and Panos J Antsaklis. Stability and stabilizability of switched linear systems: a survey of recent results. IEEE Transactions on Automatic control, 54(2):308-322, 2009.

[45] Matheus Souza, André R Fioravanti, and Robert N Shorten. On analysis and design of discrete-time constrained switched systems. International Journal of Control, 91(2):437-452, 2018.

[46] Vijay Gupta, Richard M Murray, Ling Shi, and Bruno Sinopoli. Networked sensing, estimation and control systems.

[47] Oswaldo LV Costa, Marcelo D Fragoso, and Ricardo P Marques. Discrete-time Markov jump linear systems. Springer Science \& Business Media, 2006.

[48] Fernando D Bianchi, Ricardo S Sánchez-Peña, and Marc Guadayol. Gain scheduled control based on high fidelity local wind turbine models. Renewable Energy, 37(1):233-240, 2012.

[49] Pang-Chia Chen and Jeff S Shamma. Gain-scheduled $l^{1}$-optimal control for boiler-turbine dynamics with actuator saturation. Journal of Process Control, 14(3):263-277, 2004.

[50] Fen Wu. Switching LPV control design for magnetic bearing systems. In Proceedings of the IEEE International Conference on Control Applications (CCA'01), 2001, pages 41-46. IEEE, 2001.

[51] Sigurd Skogestad and Ian Postlethwaite. Multivariable feedback control: analysis and design, volume 2. Wiley New York, 2007.

[52] Maurício C de Oliveira, Jacques Bernussou, and José C Geromel. A new discrete-time robust stability condition. Systems \& Control Letters, 37(4):261-265, 1999.

[53] Thierry Marie Guerra, Raymundo Márquez, Alexandre Kruszewski, and Miguel Bernal. $H_{\infty}$ LMI-based observer design for nonlinear systems via Takagi-Sugeno models with unmeasured premise variables. IEEE Transactions on Fuzzy Systems, 26(3):1498-1509, 2018.

[54] Johan Lofberg. YALMIP: A toolbox for modeling and optimization in MATLAB. In Proceedings of the IEEE International Symposium on Computer Aided Control Systems Design, 2004, pages 284-289. IEEE, 2004.

[55] ApS Mosek. The MOSEK optimization toolbox for MATLAB manual, 2015.

[56] David Schlipf, Dominik Johannes Schlipf, and Martin Kühn. Nonlinear model predictive control of wind turbines using LIDAR. Wind Energy, 16(7):1107-1129, 2013.

[57] Carsten Scherer, Pascal Gahinet, and Mahmoud Chilali. Multiobjective output-feedback control via LMI optimization. IEEE Transactions on Automatic Control, 42(7):896-911, 1997.

[58] Ke Zhang, Bin Jiang, and Peng Shi. Observer-based integrated robust fault estimation and accommodation design for discrete-time systems. International Journal of Control, 83(6):1167-1181, 2010. 


\section{A Wind Speed and Pitch Reference Estimation Results in the WF Benchmark}

The WF benchmark provides the real effective WS signals $\nu^{i, j}$ for numerical validations of the wind propagation schemes. The mean WS signals $\nu^{i}$ are not available.

Fig.A.1 shows the WS estimation results for the WTs $(i=1, j=1),(i=2, j=1)$ and $(i=3, j=1)$ of the $0^{\circ}$ layout. Similar estimation results are obtained for the WTs in the columns $j=2$ and $j=3$ because the propagation error is the same for all the WTs in a row (see the errors in the second part of Fig.A.1). For its part, Fig.A.2 compares the results obtained when filtering and not filtering the prorogated mean WS. One can verify that the low-pass filter in Remark 3 leads to smoother errors in the same order of magnitude as the errors of the non-filtered estimated WSs.

In the first part of Fig.A.3 we show the turbulence of the WTs in the row $i=1$, which we compute as $\tilde{\nu}_{t}^{i, j}=$ $\nu^{i, j}-1 / 3 \sum_{j=1}^{3} \nu^{i, j}$. For each sample $k$ of the time series, we depict the pair $\left(\hat{\nu}^{i}, \tilde{\nu}_{t}^{i, j}\right)$. The numerical simulations validate the bound $\left|\tilde{\nu}_{t}^{i, j}\right| \leq \lambda_{t}$ with $\lambda_{t}=3 \sigma_{t}$, which is depicted through a dashed gray line. In the second part of the figure, we show the total error of the WTs in the row $i=1$, which we compute as $\tilde{\nu}^{i, j}=\nu^{i, j}-\hat{\nu}^{i}$. For each sample $k$ of the time series, we depict the pair $\left(\hat{\nu}^{i}, \tilde{\nu}^{i, j}\right)$. Provided that the mean WS signals $\nu^{i}$ are not available, we cannot compute the wind propagation error as $\tilde{\nu}_{p}^{i}=\nu^{i}-\hat{\nu}^{i}$. Thus, we conservatively bound the wind propagation error with the 99-th percentile of the total error $\tilde{\nu}^{i, j}$, which is depicted through a dashed gray line.

Remark 11. From (10), we deduce that the propagation error is larger for higher WSs. Thus, one could consider the bound $\left|\tilde{\nu}_{p}^{i}\right| \leq \lambda_{p}^{i}\left(\tilde{\nu}_{p}^{i}\right)$, where $\lambda_{p}^{i}\left(\hat{\nu}^{i}\right)$ would depend not only on the row $i$ but also on the estimated mean WS.

As shown in Fig.A.3 (including the 99-th percentiles of different intervals), we can neglect the dependence of the bounds on the estimated mean WS.

$i=1$
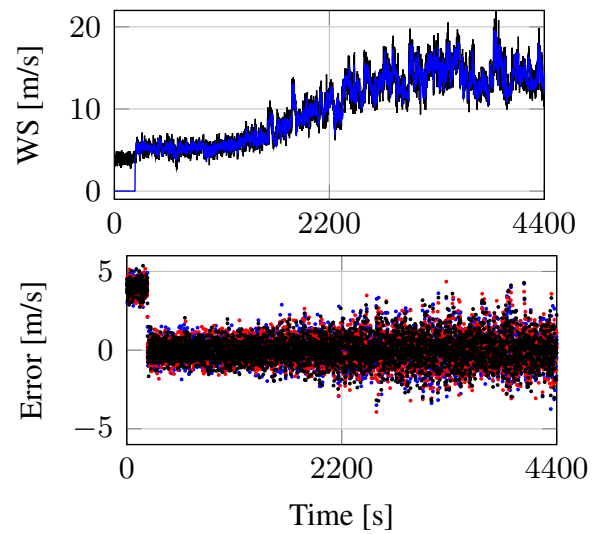

$i=2$
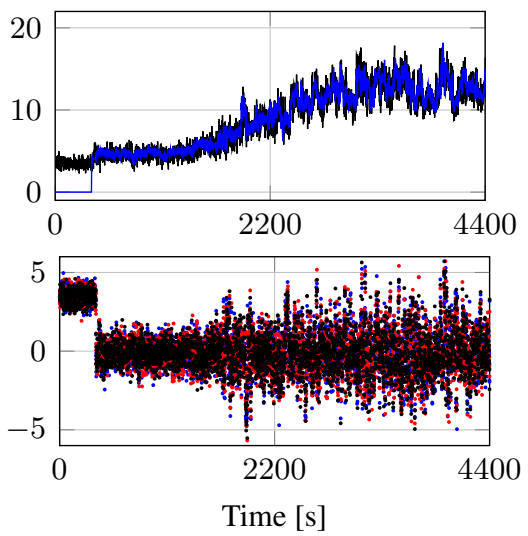

$i=3$
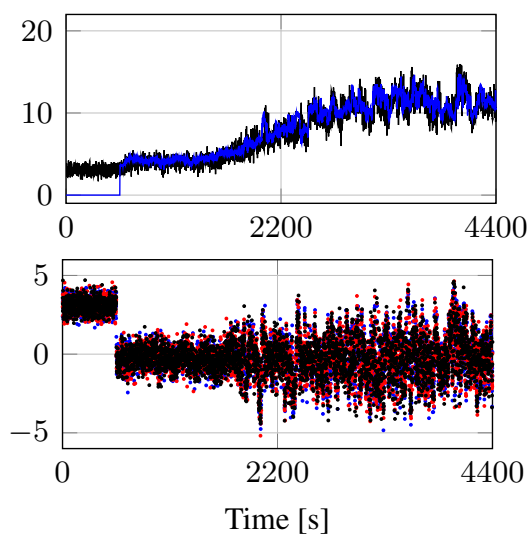

Real $\bullet j=1 \bullet j=2 \bullet j=3$

Figure A.1. Wind speed (WS) estimation for the $0^{\circ}$ layout.

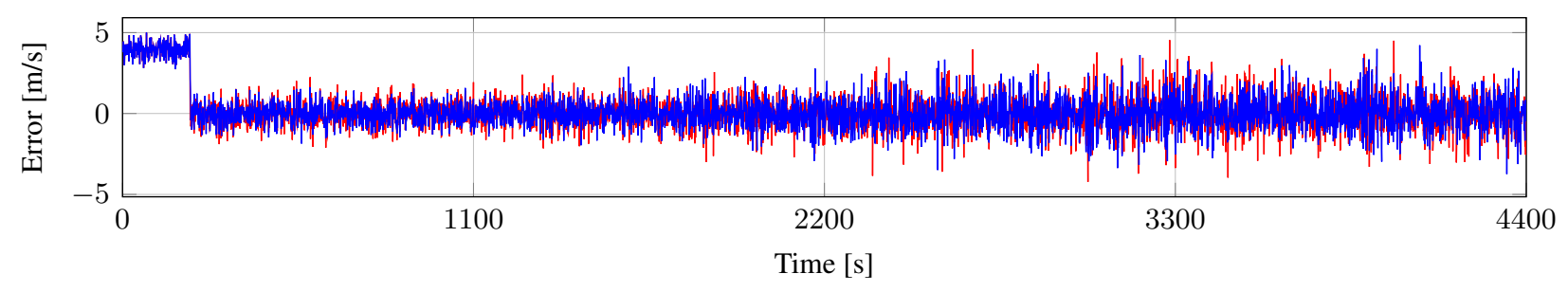

Non-filtered propagation scheme — Filtered propagation scheme

Figure A.2. Comparison of the WS estimation error with non-filtered and filtered estimated mean WS $\left(0^{\circ}\right.$ layout, $\left.i=1, j=1\right)$. 

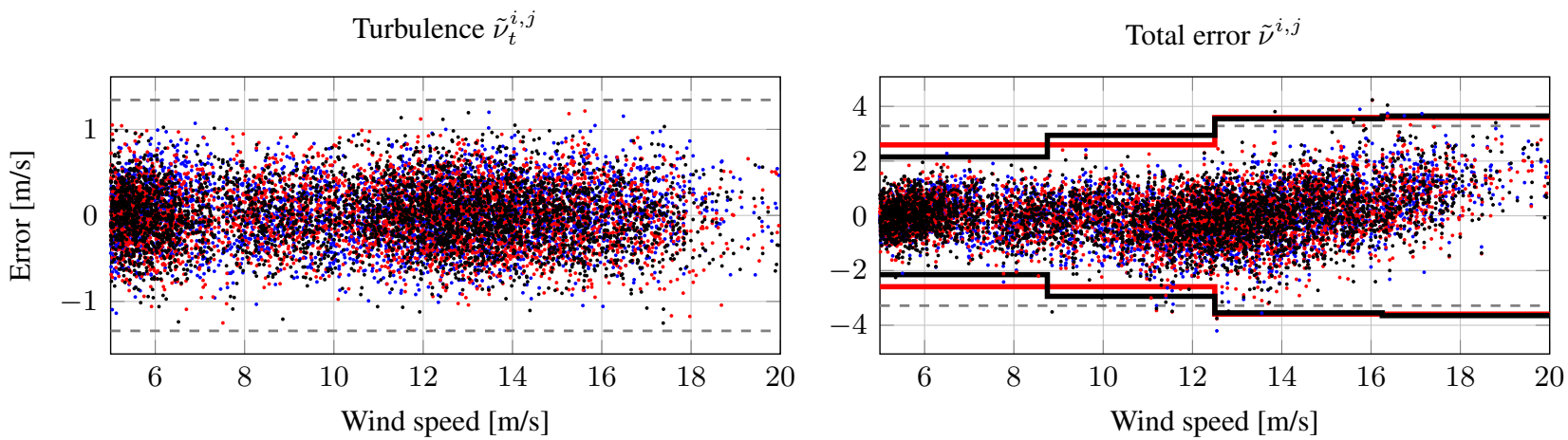

$$
\begin{aligned}
& \text { - } j=1 \quad \bullet j=2 \quad \bullet \quad j=3 \\
& \text { - - } N_{v}=1-N_{v}=2-N_{v}=3
\end{aligned}
$$

Figure A.3. Wind speed estimation error due to the turbulence and to the propagation model mismatch $\left(0^{\circ}\right.$ layout, $\left.i=1\right)$.
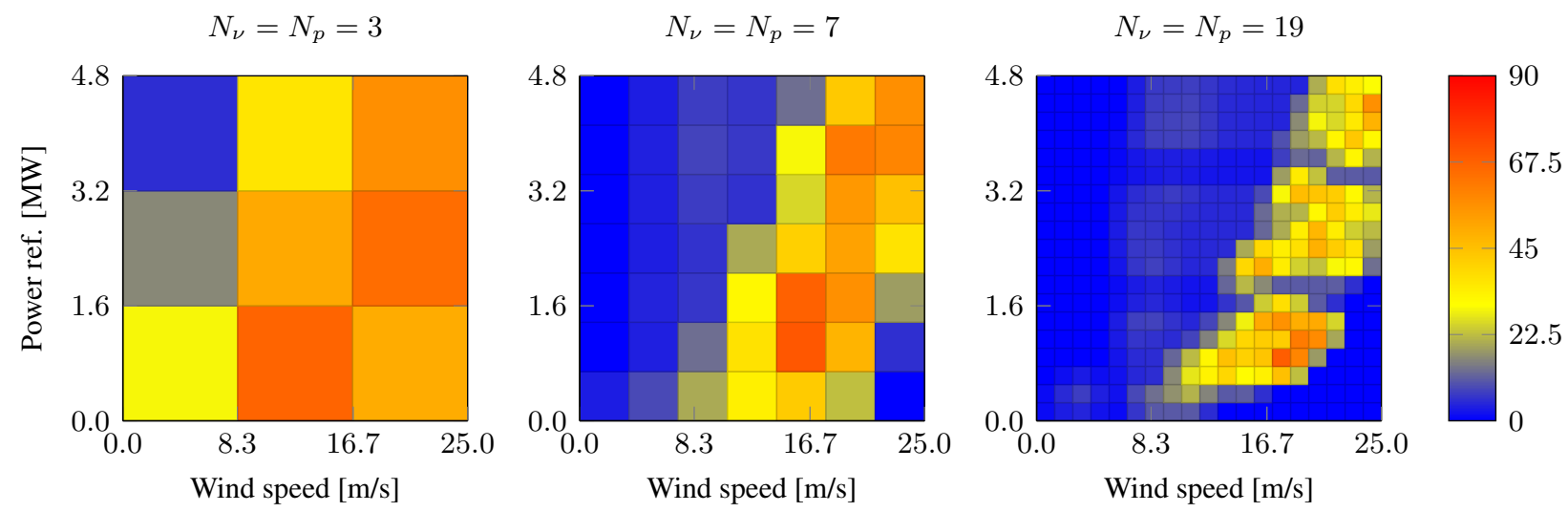

Figure A.4. Bounds of the pitch estimation error due to the propagation model mismatch $\left[{ }^{\circ}\right]\left(0^{\circ}\right.$ layout, $\left.i=1\right)$.

Once the bounds $\left|\tilde{\nu}_{p}^{i}\right| \leq \lambda_{p}^{i}$ and $\left|\tilde{\nu}_{t}^{i, j}\right| \leq \lambda_{t}$ have been computed, we determine the bounds of the errors of the pitch reference estimation. In the following, we include the computation of the bounds of the errors $\tilde{u}^{i}$ (which depend on $\lambda_{p}^{i}$ ). A similar procedure is used to determine the bounds of the errors $\tilde{u}^{i, j}$ (which depend on $\lambda_{p}^{i}$ and $\lambda_{t}$ ). Fig.A.4 includes the values of the bounds $\lambda_{1, q}^{i}\left(\left|\tilde{u}^{i}\right| \leq \lambda_{1, q}^{i}\right)$ verifying (20). We include the results obtained when using three different numbers of intervals $N_{\nu}$ and $N_{p}$. As the number of griding intervals increases, the points in the set $\Theta$ associated to low pitch estimation errors increase. This enhances better FE and FD performances at the cost of heavier computational times. Thus, we choose the intermediate solution defined by $N_{\nu}=N_{p}=7$.

\section{B Convex Formulation of the Observer Design Optimization Problem}

The observer design optimization problem (35) can be reformulated as a multiobjective convex optimization problem as follows.

$$
\begin{array}{lll}
\operatorname{minimize} & \sum_{q=1}^{N_{\theta}} \sum_{j=1}^{n_{j}}\left(\gamma_{w, q}^{j} \sigma_{w}^{2}+\gamma_{p, q}^{j}\left(\lambda_{1, q}^{i}\right)^{2}+\gamma_{t, q}^{j}\left(\lambda_{2, q}^{i}\right)^{2}\right) \\
\text { subject to } & \Xi_{q, p}^{1} \succeq 0, \quad \forall(q, p) \in\left\{1, \ldots N_{\theta}\right\} \times\left\{1, \ldots N_{\theta}\right\} \\
& \Xi_{q, j}^{\{2,3,4,5,6,7\}} \succeq 0, \quad \forall(q, j) \in\left\{1, \ldots N_{\theta}\right\} \times\left\{1, \ldots n_{j}\right\}
\end{array}
$$


along the full-rank matrices $Q_{q}, K_{q}, X_{q}$; symmetric matrices $P_{q, j}, R_{q, j}, S_{q, j}, T_{q, j}, V_{q, j}$; and scalars $\gamma_{q, j}^{w}, \gamma_{q, j}^{p}$ and $\gamma_{q, j}^{t}$ for $q=1, \ldots, N_{\theta}$ and $j=1, \ldots, n_{j}$. The LMIs in (50) are defined as ${ }^{10}$

$$
\begin{aligned}
& \Xi_{q, p}^{1}=\left[\begin{array}{cc}
Q_{q}+Q_{q}^{T}-P_{p} & \Xi_{q} \\
\star & P_{q}
\end{array}\right] \\
& \Xi_{q, j}^{2}=\left[\begin{array}{ccc}
Q_{q}+Q_{q}^{T}-R_{q, j} & \Xi_{q} & 0 \\
\star & R_{q, j} & \Psi_{q}^{j} \\
\star & \star & I
\end{array}\right] \quad \Xi_{q, j}^{3}=\left[\begin{array}{cc}
Q_{q}+Q_{q}^{T}-R_{q, j} & Q_{q} D \\
\star & \Gamma_{q}^{j}
\end{array}\right] \\
& \Xi_{q, j}^{4}=\left[\begin{array}{ccc}
Q_{q}+Q_{q}^{T}-S_{q, j} & \Xi_{q} & 0 \\
\star & S_{q, j} & \Psi_{q}^{j} \\
\star & \star & I
\end{array}\right] \quad \Xi_{q, j}^{5}=\left[\begin{array}{ccc}
Q_{q}+Q_{q}^{T}-S_{q, j} & X_{q} & 0 \\
\star & \gamma_{w, q}^{j} I & \left(F^{j} K_{q}\right)^{T} \\
\star & \star & I
\end{array}\right] \\
& \Xi_{q, j}^{6}=\left[\begin{array}{cccc}
Q_{q}+Q_{q}^{T}-T_{q, j} & \Xi_{q} & Q_{q} E & 0 \\
\star & T_{q, j} & 0 & \Psi_{q}^{j} \\
\star & \star & \gamma_{p, q}^{j} I & 0 \\
\star & \star & \star & I
\end{array}\right] \quad \Xi_{q, j}^{7}=\left[\begin{array}{cccc}
Q_{q}+Q_{q}^{T}-V_{q, j} & \Xi_{q} & Q_{q} F & 0 \\
\star & V_{q, j} & 0 & \Psi_{q}^{j} \\
\star & \star & \gamma_{t, q}^{j} I & 0 \\
\star & \star & \star & I
\end{array}\right]
\end{aligned}
$$

with $\Xi_{q}=Q_{q} A-X_{q} C, \Psi_{q}^{j}=\left(F^{j} R-F^{j} K_{q} C_{q}\right)^{T}$ and

$$
\Gamma_{q}^{j}=\left[\begin{array}{ccc}
\bar{\gamma}_{1}^{j} & 0 & 0 \\
0 & \ddots & 0 \\
0 & 0 & \bar{\gamma}_{n_{j}}^{j}
\end{array}\right]
$$

The gains $L_{q}$ are then defined as $L_{q}=Q_{q}^{-1} X_{q}$. The proofs associated to this procedure can be found in reference works $[43,52,57,58]$, where it is shown how to translate a multiobjective optimization problem into a convex optimization problem based on LMIs by means of introducing of slack variables, here named after $\left\{Q_{q}\right\}_{q \in\left\{1, \ldots, N_{\theta}\right\}}$.

\footnotetext{
${ }^{10}$ The symbol $\star$ in a block matrix denotes the blocks induced by symmetry.
} 